\title{
Inversion of a topological domain leads to restricted changes in its gene expression and affects inter-domain communication
}

\author{
Authors \\ Rafael Galupa ${ }^{1 \#^{*}}$, Christel Picard ${ }^{1 \#}$, Nicolas Servant ${ }^{2,3}$, Elphège Pierre Nora ${ }^{1 \neq}$, Yinxiu Zhan ${ }^{4}$ \\ ${ }^{5}$, Joke van Bemmel ${ }^{1}$, Fatima El Marjou ${ }^{6}$, Colin Johanneau ${ }^{6}$, Maud Borensztein ${ }^{1 \pm}$, Katia \\ Ancelin $^{1}$, Luca Giorgetti ${ }^{4}$ and Edith Heard ${ }^{1,7^{*}}$
}

\section{Affiliations}

${ }^{1}$ Mammalian Developmental Epigenetics Group, Genetics and Developmental Biology Unit, Institut Curie, PSL Research University, CNRS UMR3215, INSERM U934, Paris, France.

2 Bioinformatics, Biostatistics, Epidemiology and Computational Systems Unit, Institut Curie, PSL Research University, INSERM U900, Paris, France.

${ }^{3}$ MINES ParisTech, PSL Research University, CBIO-Centre for Computational Biology, Paris, France.

${ }^{4}$ Friedrich Miescher Institute for Biomedical Research, Basel, Switzerland.

${ }^{5}$ University of Basel, Switzerland.

${ }^{6}$ Transgenesis Facility, Institut Curie, Paris, France.

${ }^{7}$ Collège de France, Paris, France.

${ }^{\#}$ Equal contribution

* Correspondence to: rafael.galupa@embl.de and edith.heard@embl.org; current address: European Molecular Biology Laboratory, Heidelberg, Germany

${ }^{*}$ Current address: Cardiovascular Research Institute, University of California San Francisco, San Francisco, CA, USA; Department of Biochemistry and Biophysics, University of California San Francisco, San Francisco, CA, USA

${ }^{ \pm}$Current address: IGMM, Univ Montpellier, CNRS, Montpellier, France

\begin{abstract}
The interplay between the topological organization of the genome and the regulation of gene expression remains unclear. Depletion of molecular factors underlying genome topology, such as CTCF and cohesin, leads to modest alterations in gene expression, while genomic
\end{abstract}


rearrangements involving boundaries of topologically associating domains (TADs) disrupt normal gene expression and can lead to pathological phenotypes. Here we inverted an almost entire TAD $(245 \mathrm{~kb}$ out of $300 \mathrm{~kb})$ within the $X$-inactivation centre (Xic), leaving its boundaries intact. This led to a significant rearrangement of topological contacts within the $\mathrm{TAD}$, mostly in accordance to the orientation of underlying CTCF binding sites but suggesting heterogeneity in the "contact" potential of different CTCF sites. The inversion also led to increased contact insulation with the neighbouring TAD. Expression of most genes within the inverted TAD remained unaffected in mouse embryonic stem cells and during differentiation. Interestingly, expression in the neighbouring TAD of the noncoding transcript Xist, which controls $\mathrm{X}$-chromosome inactivation, was ectopically upregulated. The same inversion in mouse embryos led to a bias in Xist expression, but X-inactivation choice ratios did not significantly deviate from wild type. Smaller deletions and inversions of specific clusters of CTCF sites within the TAD led to similar results: rearrangement of contacts, limited changes in local gene expression but significant changes in Xist expression. Our study suggests that the wiring of regulatory interactions within a TAD can influence the expression of genes in neighbouring TADs, highlighting the existence of mechanisms for inter-TAD communication.

\section{Introduction}

The three-dimensional folding of the genome has been increasingly recognised as an essential component for our understanding of gene regulation (Dekker and Mirny, 2016; McCord et al., 2020). Chromosome conformation capture techniques (Denker and de Laat, 2016) have unravelled a complex hierarchy of structural layers that organise mammalian chromosomes, composed of domains of high frequency contacts (Zhan et al., 2017). At the sub-megabase level, these domains are generally designated topologically associating domains (TADs) (Dixon et al., 2012; Nora et al., 2012) and are well conserved across species and invariant across cell types (Dekker and Heard, 2015). The dynamics of the formation and maintenance of TADs and their boundaries during development and each cell cycle remain under investigation (Szabo et al., 2019) but seem to depend on the interplay between the architectural proteins cohesin and the zinc finger protein CTCF (Fudenberg et al., 2016; Haarhuis et al., 2017; Nora et al., 2017; Rao et al., 2017; Sanborn et al., 2015; Schwarzer et al., 2017; Wutz et al., 2017). Enriched at boundaries between TADs (Dixon et al., 2012; Phillips-Cremins et al., 2013), CTCF is required for chromatin loops observed between CTCF sites and for the organisation and insulation of most TADs (Nora et al., 2017). Remarkably, CTCF-mediated contacts mostly occur between CTCF sites for which the CTCF motifs lie in a convergent orientation (Rao et al., 2014a; Tang et al., 2015a), and the contacts depend on the orientation of the motifs: altering the orientation of a CTCF site can disrupt a loop and lead to the formation of new ones (de Wit et al., 2015; Guo et al., 2015; Sanborn et al., 2015).

TADs are thought to instruct gene regulatory landscapes, allowing promoters and their regulatory elements to meet often and lead to a more efficient transcriptional output 
(Symmons et al., 2016). Accordingly, TADs represent the folding scale at which promoterenhancer interactions and gene co-regulation are maximised (Zhan et al., 2017). The communication between promoters and enhancers is generally assumed to rely on chromatin looping, and long-range contacts within TADs can be quite dynamic during processes that involve rewiring of the regulatory networks, such as differentiation (Dixon et al., 2015). However, the interplay between such topological organization and the regulation of gene expression remains unclear. Loss of TADs upon depletion of CTCF or cohesin leads to relatively small effects on gene expression (Nora et al., 2017; Rao et al., 2017; Schwarzer et al., 2017; Wutz et al., 2017), and genomic rearrangements involving mammalian TADs and their boundaries can have either very modest effects (Amândio et al., 2020; Despang et al., 2019; Paliou et al., 2019; Rodríguez-Carballo et al., 2017; Williamson et al., 2019) or disrupt normal gene expression and underlie pathological phenotypes (Flavahan et al., 2016; Franke et al., 2016; Hnisz et al., 2016; Lupiáñez et al., 2015).

Here we set out to explore the relationship between TAD organisation and transcriptional regulation at a critical developmental regulatory landscape, the mouse $X$-inactivation centre (Xic). The Xic is the master regulator for the initiation of X-chromosome inactivation in female placental mammals (Augui et al., 2011a; Rastan and Brown, 1990), harbouring the noncoding RNA Xist locus and the regulatory elements necessary for its female-specific developmental control. Xist is repressed in mouse embryonic stem cells (mESCs) and becomes upregulated from one of the two X-chromosomes in females upon exit of the pluripotent state, leading to random X-inactivation. This upregulation depends on Xist cisregulatory landscape (Heard et al., 1999), the full extent of which is still undefined - it is, however, partitioned in at least two TADs, with the Xist locus lying close to the boundary between them (Fig. 1A) (Nora et al., 2012). The TAD in which the Xist promoter is included (here referred to as Xist-TAD) contains some of Xist positive regulators (Augui et al., 2007; Barakat et al., 2011, 2014; Furlan et al., 2018; Gontan et al., 2012; Jonkers et al., 2009; Tian et al., 2010), while the adjacent TAD (here referred to as Tsix-TAD) contains the promoter of Tsix, the antisense transcription unit to Xist that blocks its upregulation (Lee and Lu, 1999; Luikenhuis et al., 2001; Stavropoulos et al., 2001) as well as other elements that act as a cisrepressors of Xist (such as Linx and Xite, see more below).

To investigate how the topological organisation of the Tsix-TAD impacts the regulation of genes therein, and in the neighbouring Xist-TAD, we generated a mutant allele in mESCs and in mice with an inversion of almost the entire Tsix-TAD $(245 \mathrm{~kb}$ out of $300 \mathrm{~kb})$. We found that rewiring the Tsix-TAD structural landscape led to the formation of new chromatin contacts within the TAD, generally following the folding principles determined by the orientations of CTCF motifs. These topological alterations were accompanied by changes in gene expression of two out of seven genes within the TAD in differentiating mESCs. Interestingly, we found that the expression of Xist, in the neighbouring TAD, was ectopically upregulated, suggesting that inter-TAD communication was affected 


\section{Results}

Generating a genomic inversion encompassing the Tsix-TAD (245kb-INV)

The Tsix-TAD harbours three hotspots of physical contacts (Fig. 1A), involving three different loci: (1) the Xite element, a proximal enhancer of Tsix (Ogawa and Lee, 2003) also involved in the position and insulation of the boundary between the Tsix- and Xist-TADs (van Bemmel et al., 2019); (2) the noncoding Linx locus, which harbours two cis-regulatory elements involved in controlling Xist expression (Galupa et al., 2020); and (3) Chic1, previously implicated in the maintenance of the organisation of the Tsix-TAD (Giorgetti et al., 2014). Each of these loci harbours a set of CTCF sites involved in mediating the observed physical contacts, and within each locus, most CTCF motifs present the same orientation (Fig. 1A). Sites within Linx are "convergently oriented" towards those within Chic1 or Xite, the preferred orientation to form chromatin loops (Rao et al., 2014a; Tang et al., 2015b). Contacts between Chic1 and Xite (the CTCF motifs of which occur in a "tandem") are also observed (Fig. 1A). The contacts between these three loci might occur in pairwise fashion and/or simultaneously; physical modelling suggests that all conformations are possible (Giorgetti et al., 2014) and deletions of the CTCF binding sites in either Xite (van Bemmel et al., 2019) or Linx (Galupa et al., 2020) show that contacts between the two remaining loci still occur.

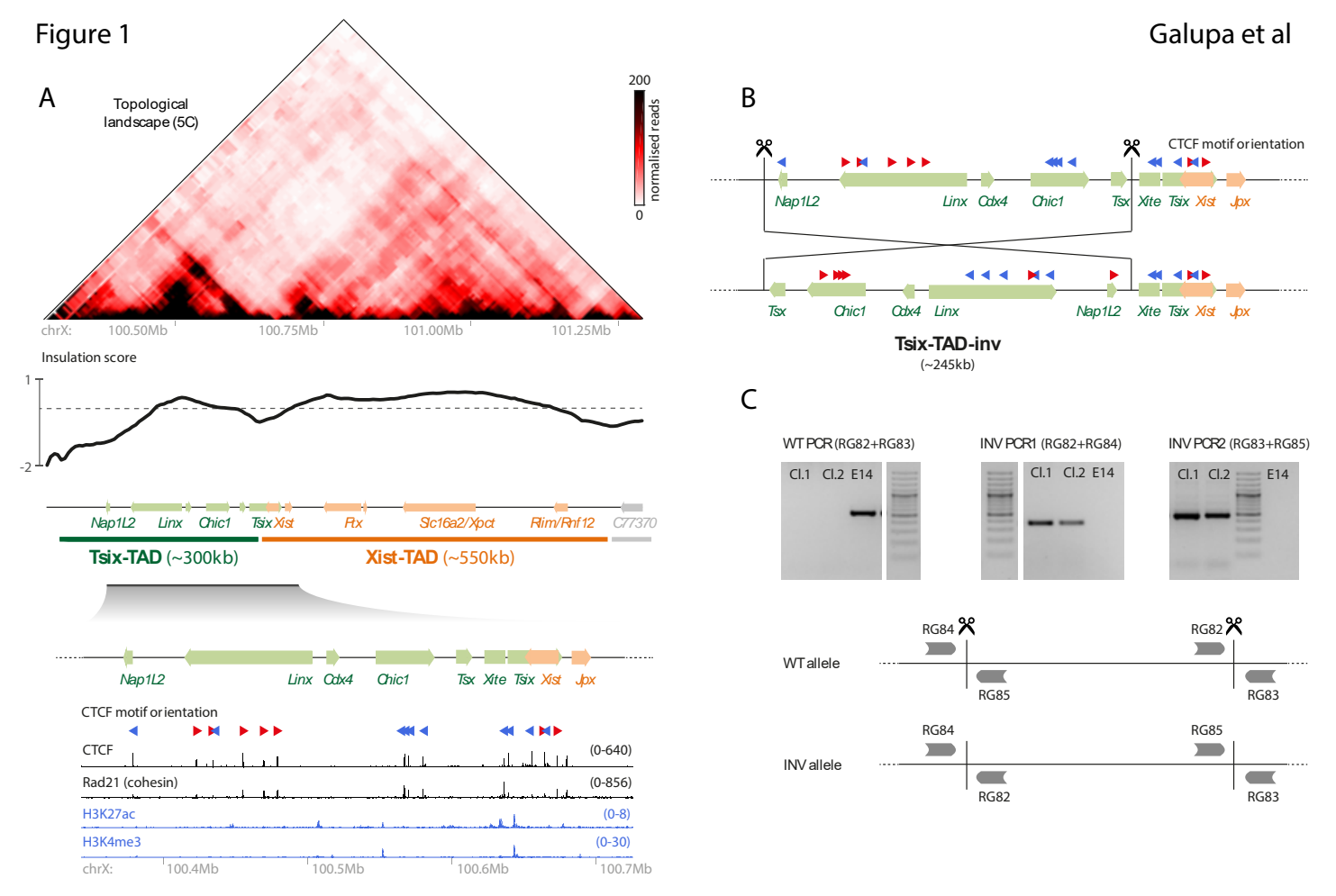


We wondered whether this complex topological organization might be critical to ensure correct communication between the surrounding cis-regulatory elements (such as those within Xite and Linx) and their targets, and therefore ensure appropriate gene expression of Tsix and Xist and correct patterns of X-inactivation. Using a CRISPR/Cas9 editing approach in male mESCs, which carry a single $X$ chromosome, we targeted a $\sim 245 \mathrm{~kb}$ region encompassing all loci within the Tsix-TAD, including the CTCF clusters within Linx and Chic1, but excluding Xite and Tsix (Fig. 1B). We decided not to include Xite in the inversion because (i) Xite is already known to influence Xist expression (via Tsix), and (ii) if Xite was inverted along with the rest of the TAD, the relative CTCF orientations between Xite, Linx and Chic1 would not have changed. The targeted region does not involve either of the two boundaries of the TAD. We successfully generated two clones harbouring an inversion allele (245kb-INV) (Fig. 1C). This genomic inversion swaps the orientations of all CTCF motifs therein relative to those outside of the inverted region, in particular for Linx and Chic1 (Fig. 1B), and is therefore expected to lead to the formation of new contacts within the TAD.

$245 \mathrm{~kb}$-INV leads to rearrangement of contacts within the TAD and increased insulation with neighbouring $T A D$

To assess the topological organisation of the $245 \mathrm{~kb}-\mathrm{INV}$ allele, we performed carbon-copy chromosome conformation capture (5C) on the Xic (Dostie et al., 2006; Nora et al., 2012) for mutant and control mESCs (Fig. 2A). 5C analysis revealed that three hotspots of contacts can still be observed in the Tsix-TAD on the $245 \mathrm{~kb}-\mathrm{INV}$ allele (Fig. 2B; please note that the 5C map is shown after "correction" of the new genomic sequences in the inverted allele). These involve the same three loci as in control cells: in its new position, the Chic1 CTCF cluster is able to establish contacts with Linx and with Xite (Fig. 2B) and Linx and Xite, with CTCF sites in "tandem" orientation in the $245 \mathrm{~kb}-\mathrm{INV}$ allele, also interact together (like Chic1 and Xite do in control cells) (Fig. 2B). Inverting the Linx and Chic1 CTCF clusters simultaneously seems therefore to lead to new but similar hotspots of physical contacts within the Tsix-TAD compared to control. This could have been expected given that the overall distribution and orientation of CTCF sites within the TAD remains similar between the wild type and the inverted alleles (Fig. 1B). In other words, the Chic1 CTCF cluster on the inverted allele occupies an equivalent position to Linx on the wild type allele, and viceversa. Therefore, the $245 \mathrm{~kb}$ inversion can lead to the formation of similar loops within the Tsix-TAD compared to wild type - yet, involving different cis-regulatory elements.

Nevertheless, we also noticed some significant differences in the topology of the "inverted" Tsix-TAD. Increased contacts could be observed upstream of the inverted region, corresponding to contacts stemming from the Linx CTCF cluster in its new position (Fig. 2B, bottom, black arrow, red region in the differential map; this region shows no particular chromatin signatures, such as CTCF binding or active chromatin marks). This suggests a different "strength" for the Linx and Chic1 CTCF clusters: in the inverted allele, the Linx CTCF cluster strongly interacts with regions upstream of Chic1 (Fig. 2B, bottom, black arrow), while in the wild type configuration, the Chic1 CTCF cluster does not form such 
strong contacts with regions upstream of $\operatorname{Linx}$ (Fig. 2B, top, black arrow). Conversely, we also observed a localised, strong reduction in contacts (Fig. 2B, differential map, blue arrow) associated with the switch in positions between Linx and Chic1: the Linx CTCF cluster at its original position is able to form long-range contacts beyond Chic1 and Xite, with elements within the Xist-TAD (Fig. 2B, top, blue arrow). These contacts are lost (or strongly reduced) in the 245kb-INV cells (Fig. 2B, bottom and differential map, blue arrows), indicating that the Chicl CTCF cluster does not establish long-range contacts with the Xist-TAD when pisedel2in the Linx CTCF cluster position. This loss of contacts across the boufpallapy etedually extends along the whole Xist-TAD (Fig. 2C). Agaip this suggests a stronger potential for the

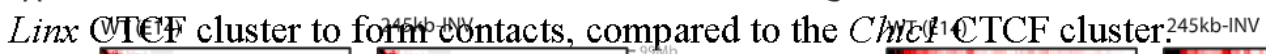

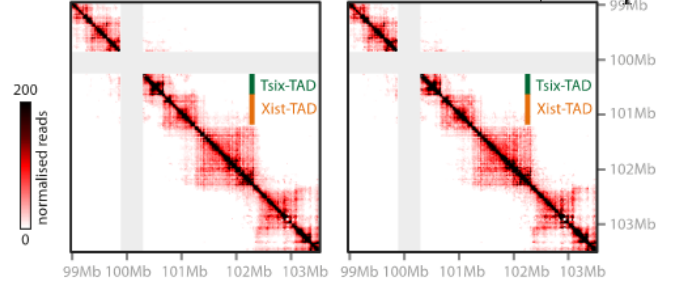

B

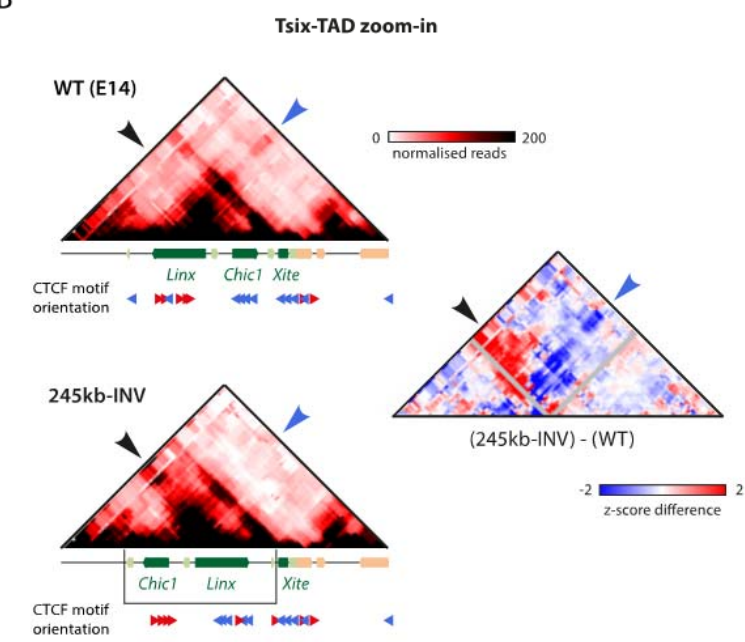

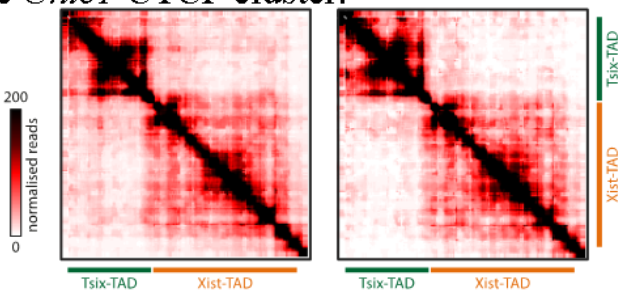

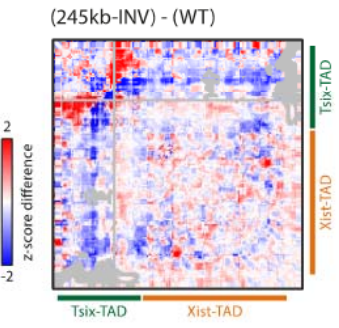

D

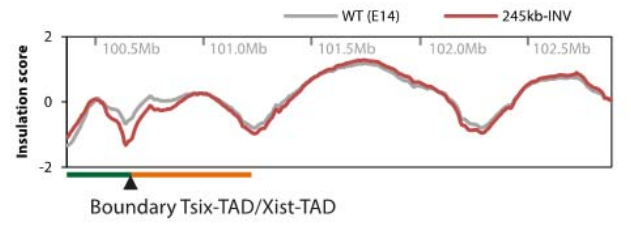

We also evaluated to which extent these topological changes had an impact in the overall insulation of the TADs. Insulation score analysis (see Methods) revealed a clear gain of insulation across the boundary between the Tsix-TAD and the Xist-TAD (Fig. 2D; lower insulation scores are reflective of increased insulation). The loss of Linx-mediated contacts across the boundary probably accounts for this increased insulation between the TADs, at least partially. In summary, the $245 \mathrm{~kb}$ inversion repositions CTCF clusters within the Tsix$\mathrm{TAD}$, leading to a reconfiguration of specific intra- and inter-TAD contacts accompanied by stronger insulation with the neighbouring Xist-TAD. 
$245 \mathrm{~kb}$-INV leads to gene expression changes within the Tsix-TAD and across the boundary

We next set out to understand if similar interaction patterns, but different wiring of sequences within the Tsix-TAD, led to any transcriptional changes. To this end, we profiled transcript levels across the Xic using digital gene expression analysis (NanoString) (Geiss et al., 2008) in control and mutant cells in the pluripotent state $(\mathrm{d} 0)$ and during early differentiation $(\mathrm{d} 0.5$ d2.5) (Fig. 3A). Expression of most genes within the Tsix-TAD and the Xist-TADs was unaffected in $245 \mathrm{~kb}$-INV cells (Fig. 3B), including that of the three loci involved in the topological alterations, Linx, Chic1 and Xite. This suggests no or limited effect of the structural alterations on the transcriptional regulation of these loci. Expression of Tsix was significantly reduced in mutant cells in the pluripotent state (d0) (Fig. 3B) but such effect did not persist consistently during differentiation. The deletion of the same region that we inverted here also led to downregulation of Tsix in mESC (Galupa et al., 2020); together with the current data, this suggests that the region contains important sequences for Tsix regulation and that this regulation depends on the orientation of the region as a whole, and might depend orfitheegrientation of individual regulatory sequences.

Galupa et al

A

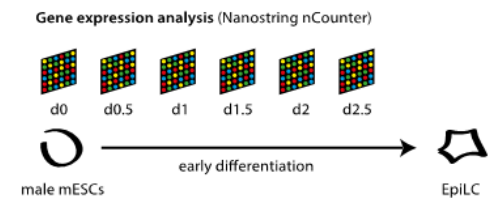

C

RNA FISH on male differentiating mESC (day 1.5) Wild type $(n=114 ; N=1)$

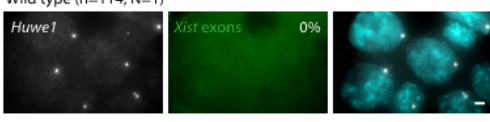

$245 \mathrm{~kb}-$ INV clone $1(\mathrm{n}=118 ; \mathrm{N}=1)$

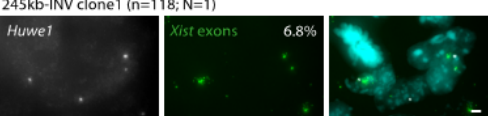
$245 \mathrm{~kb}-1 \mathrm{NV}$ clone2 $(\mathrm{n}=113 ; \mathrm{N}=1)$
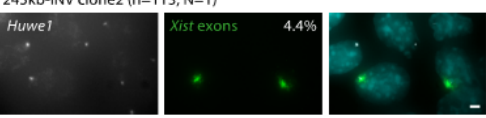
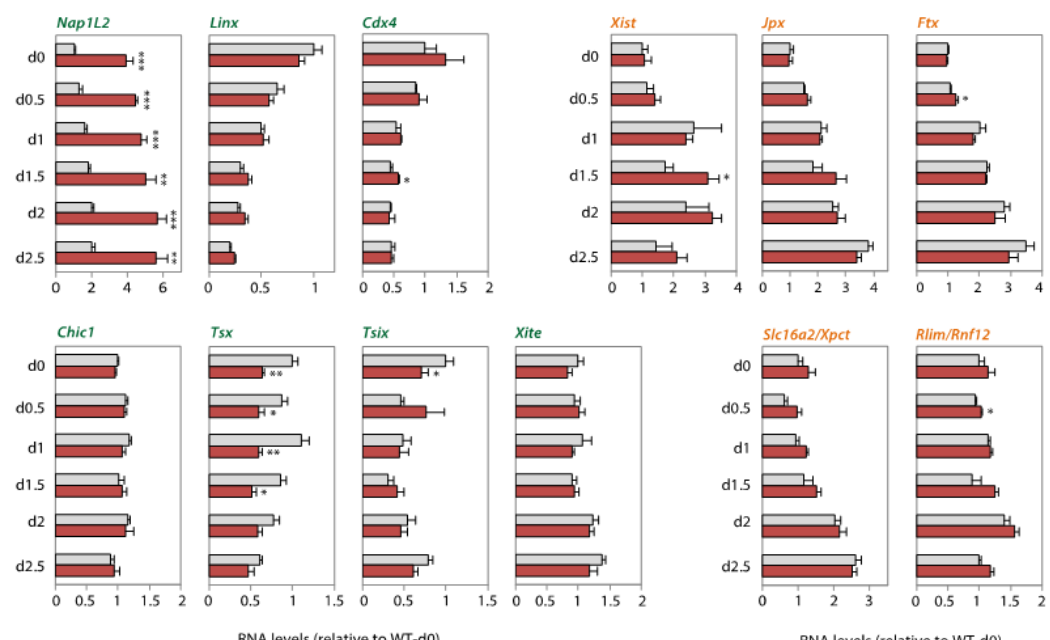

RNA levels (relative to WT-d0) 
We did notice however consistent changes during differentiation in mutant cells for two genes within the Tsix-TAD: Nap1L2, which was significantly upregulated at all time points (Fig. 3B) and Ts $x$, which was significantly downregulated (Fig. 3B). Interestingly, both genes lie at the extremities of the inverted region, and switch their relative positions in the TAD between the wild type and mutant configurations. It is likely that changes in their gene expression are associated with altered proximity to the Xite enhancer element. Since deletion of Xite leads to downregulation of TsX (van Bemmel et al., 2019), moving TsX away from Xite on the $245 \mathrm{~kb}-\mathrm{INV}$ allele could lead to its observed downregulation. Conversely, increased linear proximity of Nap1L2 to Xite could possibly underlie Nap1L2 upregulation. Changes in interaction frequencies between Xite and these two elements in the $245 \mathrm{~kb}-\mathrm{INV}$ allele do support this hypothesis, as they reflect the changes in their genomic distances (increased for Xite-Nap1L2 and decreased for Xite-TsX, compared with control, Fig. S1).

We also observed changes in expression of Xist, the long noncoding RNA locus that is regulated by the Xic to trigger the initiation of X-chromosome inactivation. Normally very low in male cells, Xist expression was slightly upregulated in the mutants at later differentiation time points ( 2 -fold at d1.5; Fig. 3B). In female cells undergoing Xinactivation, upregulation of Xist is accompanied by local accumulation of its RNA in cis, forming a so-called "Xist cloud", which can readily be detected by RNA FISH (Augui et al., 2011b). RNA FISH revealed the formation of Xist clouds in $\sim 4-7 \%$ of mutant male cells upon differentiation, which was never observed in wild type male cells (Fig. 3C). Thus, the inversion of $245 \mathrm{~kb}$ within the Tsix-TAD leads to ectopic expression of Xist, the promoter of which is located in the neighbouring TAD.

\section{Female embryos with 245kb-INV allele show higher Xist allelic imbalance}

Given the impact of the $245 \mathrm{~kb}$ inversion on Xist expression in male cells, we decided to investigate whether this was also the case in female embryos at post-implantation stages, when random X-inactivation is known to have already occurred (Rastan, 1982). To this end, we generated an equivalent $245 \mathrm{~kb}-\mathrm{INV}$ allele in mice (see Methods) and collected postimplantation heterozygous embryos. These embryos were derived from crosses between polymorphic mouse strains (Fig. 4A, 4D), which allows us to distinguish the allelic origin of transcripts. Analysis of RNA allelic ratios for Atp7a, an X-linked gene, revealed no preferential gene silencing for one or the other allele (Fig. 4B, 4E).

However, analysis of Xist RNA allelic ratios between mutant and control embryos showed slightly higher Xist allelic ratios in the mutant embryos, whether the mutant allele was inherited paternally (Fig. 4C) or maternally (Fig. 4F); this increase was statistically significant for maternal transmission $(p<0.05)$. These results are consistent with the upregulation of Xist that we observed in mutant cells (Fig. 3B-C). Thus, the $245 \mathrm{~kb}$ inversion leads to higher Xist levels in cis but this does not result in skewed patterns of X-inactivation. Of note, litter size seems to be reduced upon maternal transmission of the $245 \mathrm{~kb}-\mathrm{INV}$ allele, 
with a skewed sex ratio ( $71 \%$ females in $245 \mathrm{~kb}-\mathrm{INV}, 59 \%$ in control) suggesting that the inversion may have more phenotypic consequences.

Figure 4

A
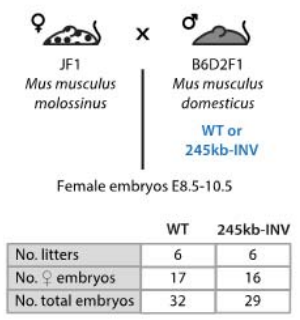

D

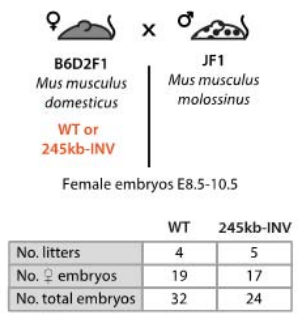

B

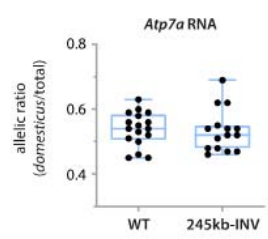

$\mathrm{E}$

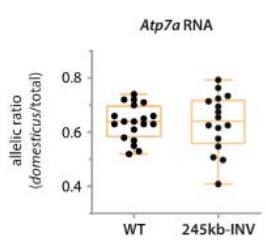

Galupa et al

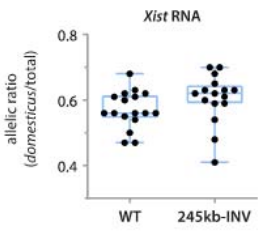

$\mathrm{F}$

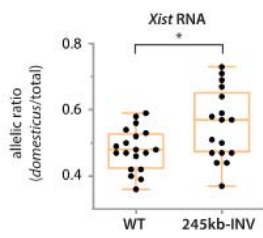

Mutating clusters of CTCF sites within Linx and Chic1 lead to changes in Xist expression

To further explore the link between the topological organization of the Tsix-TAD and Xist regulation, we decided to generate alleles with deletions and/or inversions of the clusters of CTCF sites within Linx and within Chic1. We have previously deleted a large intronic interval containing three Linx CTCF sites, in male ESCs $(\sim 51 \mathrm{~kb})$ and in mice $(\sim 25 \mathrm{~kb})$ (Galupa et al., 2020), which led to some alterations in the topological organization of the two Xic TADs but no changes in Xist expression in female embryos. We decided to test the impact of inversions of exactly the same regions, in male mESCs (Linx-51kb-INV) and in mice (Linx-25kb-INV) (Fig. 5A). 5C analysis of the Linx-51kb-INV allele revealed higher frequency of contacts between the now inverted Linx locus and regions immediately upstream (Fig. 5B, black arrowhead), and lower frequency of contacts between (inverted) Linx and Chic1, Xite and elements within the Xist-TAD (Fig. 5B-C, blue arrowhead), in agreement with the change of orientation of the three Linx CTCF sites. These results are reminiscent of what we observed for the 245kb-INV allele (Fig. 2B-C), and they support the hypothesis that loss of contacts with the Xist-TAD in the $245 \mathrm{~kb}-\mathrm{INV}$ allele is associated to inversion of the CTCF sites within Linx. Consistently, analysis of insulation scores across the TADs revealed a gain of insulation across the boundary between the Tsix-TAD and the Xist-TAD (Fig. 5D), though less pronounced than what we observed for $245 \mathrm{~kb}-\mathrm{INV}$ allele (Fig. 2D). We next analysed gene expression across the Xic for the Linx-51kb-INV mESC in the pluripotent state (d0) and during early differentiation (d0.5-d2.5); expression of Linx was significantly downregulated at some time points (Fig. 5E) but no changes were observed for Xist or Tsix 
(Fig. 5E) nor for any other locus across the Xic (data not shown). However, when we analysed Xist expression in female embryos carrying an heterozygous Linx-25kb-INV allele, we observed significantly higher expression of Xist for the inverted allele, for both paternal and maternal transmission (Fig. 5F-G), and also corresponding decrease in expression of the X-linked gene Atp $7 a$ (Fig. 5F-G), suggestive of skewed XCI compared to control. Overall, the inversion of the Linx CTCF cluster leads to similar phenotypes compared to the large $245 \mathrm{~kb}$ inversion, namely a decrease in contact ${ }_{B}$ frequency between $\operatorname{Lin} x$ and the Xist-TADs,

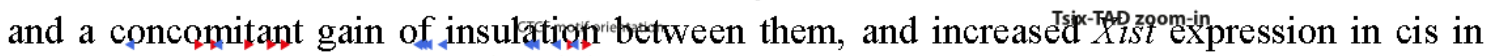

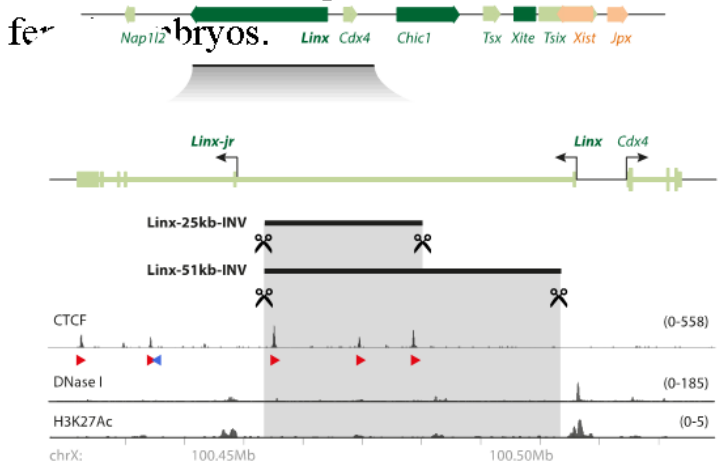

C

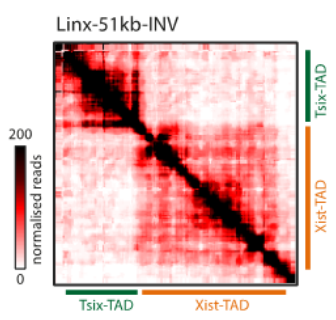

$\mathrm{E}$

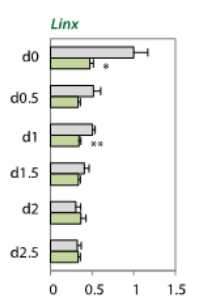

d2.5

RNA levels (relative to WT-do)

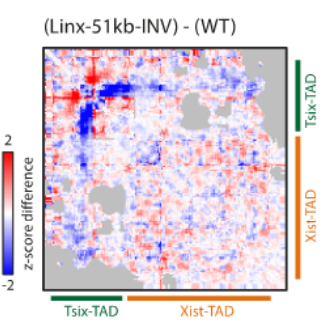

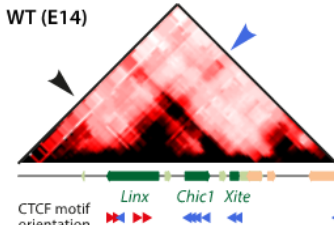

CTCF motif $\operatorname{Linx}$ Chicl Xite CTCF motif Linx $\mid$ Chicl Xite

D

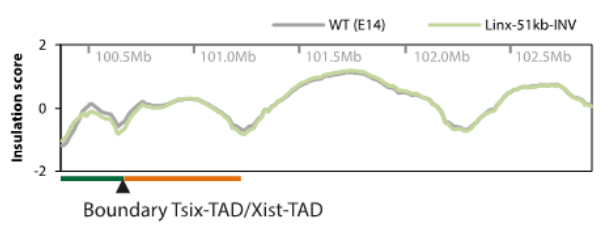

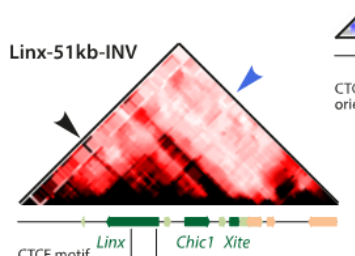

$\mathrm{F}$
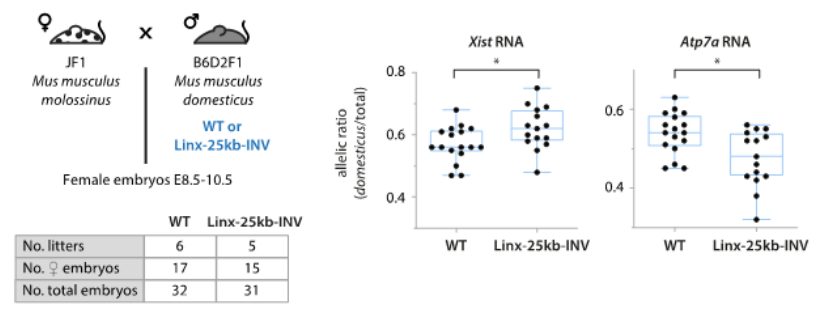

G

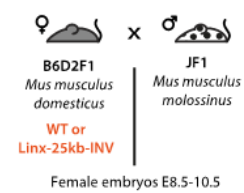

\begin{tabular}{|l|c|c|} 
& \multicolumn{1}{c}{ WT } & Linx-25kb-INV \\
\hline No. litters & 4 & 4 \\
\hline
\end{tabular}

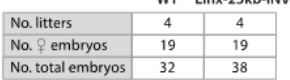

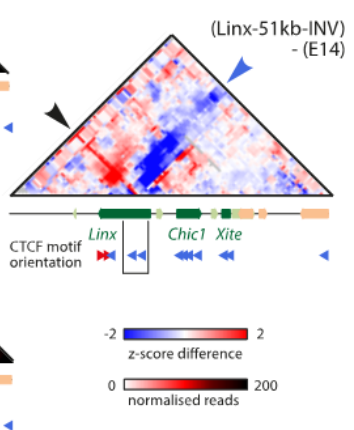

$\square W T$ (E14) $\square$ Linx-51kb-INV

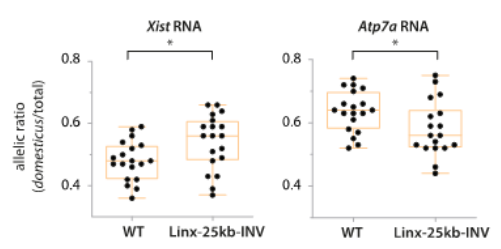


We have previously generated in male mESC a $\sim 4 \mathrm{~kb}$ deletion within Chic1 (Giorgetti et al., 2014) that encompassed two of the three CTCF binding sites present in the locus (Fig. 6A), but we did not study its impact on chromosome conformation nor on Xist expression, which we set out to do here. Differential 5C analysis between this Chic1-4kb $\Delta$ allele and wildtype revealed showed a reduction in contacts between Chic1 and Linx, and also between Chic1 and Xite (Fig. 6B), consistent with loss of the Chic1 CTCF sites. We also noted a seemingly increase in contact frequency between Linx and Xite (Fig. 6B, Fig. S1), which would be consistent with a model of competition between Chic1 and Xite CTCF sites to form loops with the CTCF sites within Linx. However, these differences in contact frequencies overall remained rather close to the "noise" levels of the $5 \mathrm{C}$ map. We wondered whether these effects would be more pronounced if the remaining CTCF binding site was also removed; thus, we generated, in male mESC, a larger deletion (Chic1-14kb $\Delta$ ) encompassing all three CTCF sites within Chic1 (Fig. 6A). We observed similar contact rearrangements within the Tsix-TAD as for Chic1-4kb $\Delta$ but more pronounced (Fig. 6C), suggesting that it is the loss of the CTCF sites that underlies the observed topological differences. To study the impact of these deletions on gene expression across the Xic, we profiled transcript levels as done previously in the pluripotent state (d0) and during early differentiation. Expression of Chic1 itself was consistently upregulated in both Chic1-4kb $\Delta$ and Chic1-14kb $\Delta$ (Fig. 6D-E); it is intriguing to think that this could potentially be linked to its now shorter length, as shorter genes have been associated to higher levels of expression (Castillo-Davis et al., 2002; Chiaromonte et al., 2003). We also observed higher expression of the gene upstream of Chic1, Cdx4: interestingly, the effects seemed to scale up with the larger deletion - in Chic1$4 \mathrm{~kb} \Delta \mathrm{mESC}$, there was a slight increase in $C d x 4$ levels across time points but not statistically significant, while in Chic $1-14 \mathrm{~kb} \Delta$ the increase was more pronounced and statistically significant for some of the time points. This effect could be connected to the removal of all CTCF sites from within the Chic1 locus, which could potentially "shield", or insulate, Cdx4 from activating influences downstream of the CTCF sites. Expression of Xist expression was also more affected in mESC containing the larger deletion: we observed a mostly consistent downregulation across all time points, but this effect was not statistically significant in this context. In female embryos, however, we did observe a statistically significant decrease in Xist expression from the deletion alleles (Fig. 6D-E), and more pronounced for the Chic1$14 \mathrm{~kb} \Delta$ allele. This suggests that the Chic1 CTCF cluster might operate to favour Xist expression in cis. These results potentially illustrate as well how loss of one additional CTCF binding site might be enough to cause stronger changes in chromosome conformation and gene expression.

Together, our results on inverting or deleting Linx and Chic1 CTCF clusters highlight the rather complex regulatory landscape within the Xic. Similar to the $245 \mathrm{~kb}$ inversion, these mutant alleles reveal how Xist is sensitive to changes involving CTCF binding sites within the neighbouring Tsix-TAD. These results also suggest that the phenotypes observed in the $245 \mathrm{~kb}-\mathrm{INV} \mathrm{mESC}$ are likely a compound of effects from changing different elements within the Tsix-TAD. 
bioRxiv preprint doi: https://doi.org/10.1101/2022.01.24.477495; this version posted January 24, 2022. The copyright holder for this preprint (which was not certified by peer review) is the author/funder, who has granted bioRxiv a license to display the preprint in perpetuity. It is made available under aCC-BY 4.0 International license.

Figure 6

\section{A}
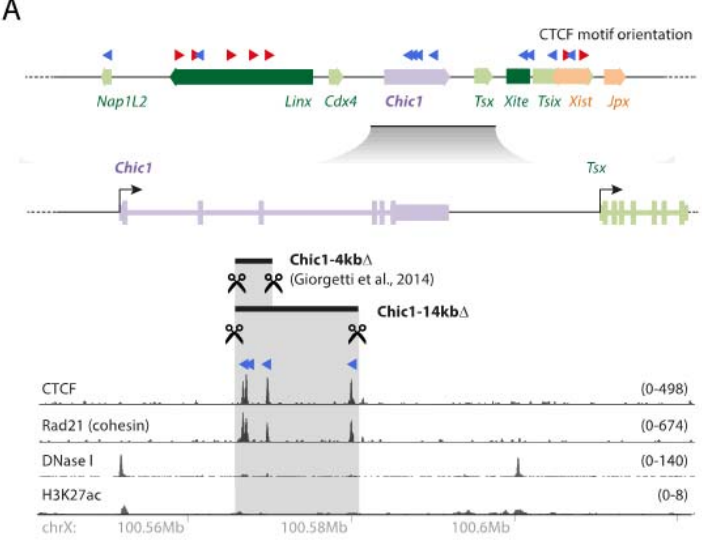

B
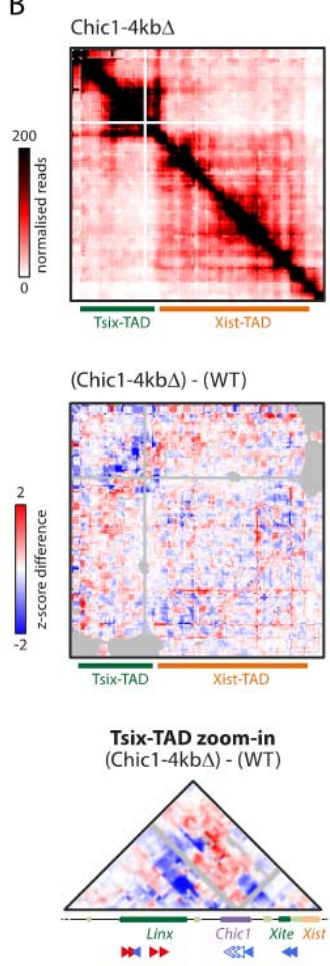

C
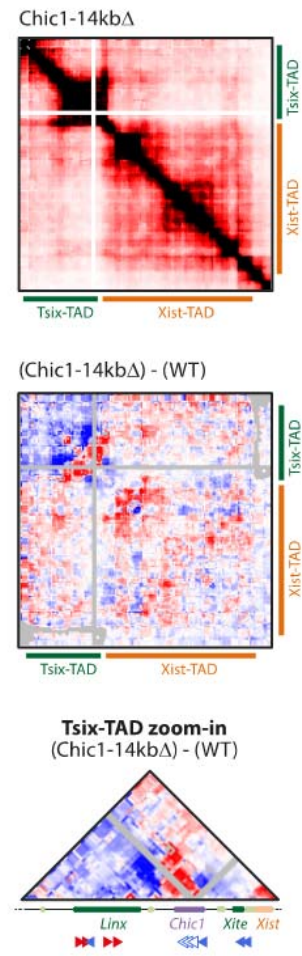

D
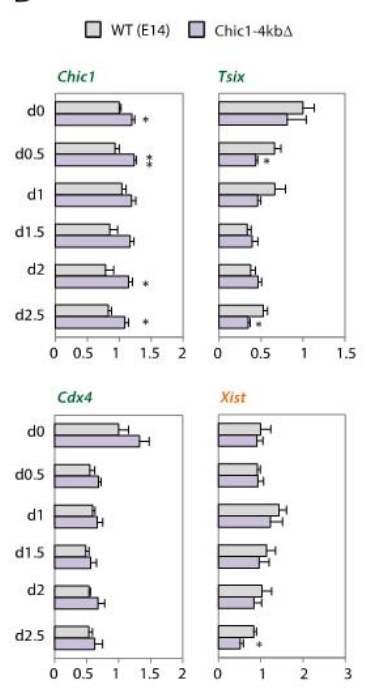

RNA levels (relative to WT-do)
Galupa and Picard et al

$\mathrm{E}$
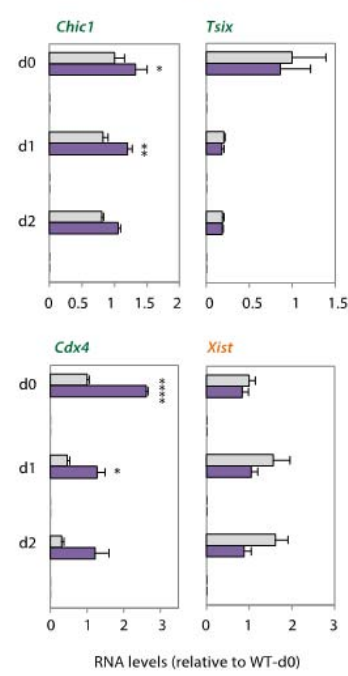

F

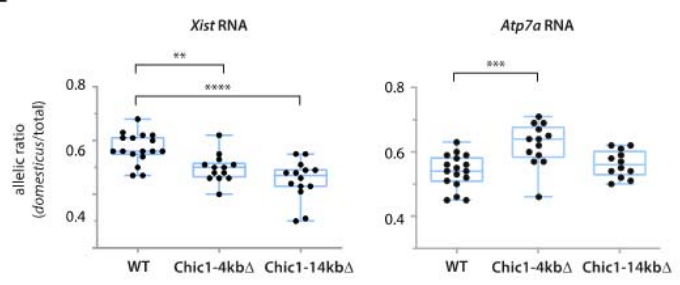

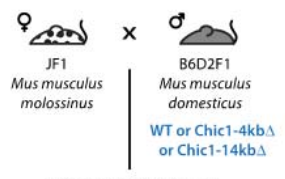

Female embryos E8.5-10.5

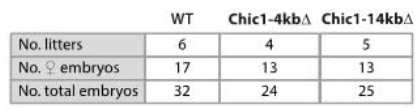

\section{Discussion}

Here we explored the structural and transcriptional consequences of inverting a large genomic region encompassing almost an entire TAD $(80 \% ; 245 \mathrm{~kb}$ out of $300 \mathrm{~kb})$. We found that this inversion led to rearrangement of contacts and to changes in expression of some 
genes within the TAD. We also observed increased contact insulation with the neighbouring TAD and ectopic upregulation of a gene in that TAD, the noncoding RNA Xist locus.

The rearrangement of contacts within the Tsix-TAD upon inverting a $245 \mathrm{~kb}$ region occurred largely as expected based on the "rules" associated with the orientation of CTCF motifs within the TAD (de Wit et al., 2015; Guo et al., 2015; Rao et al., 2014a; Sanborn et al., 2015; Tang et al., 2015b). We found that the three loci involved in the strong contacts observed in the wild type Tsix-TAD were still able to form strong contacts with each other in the "inverted" Tsix-TAD (Fig. 2). Yet, these elements could not fully replace each other in their new positions, despite similar composition in terms of number of CTCF sites and levels of CTCF binding based on published ChIP-seq data (Fig. 1). In particular, the Linx CTCF cluster seems to have a stronger potential to form contacts than the Chic1 CTCF cluster. At the same relative position within the TAD, and with the same CTCF motif orientation, these CTCF clusters show a different range of interactions, as described above (Fig. 2). These differences suggest that not all CTCF-bound sites are equally capable of mediating the same type of interactions. Little is known about what determines which CTCF sites contact with each other, and whether there could be specific affinities between sites - depending for instance on which other protein complexes are bound at each site or nearby. The ChIP-seq signal for CTCF is fairly comparable between CTCF sites within Linx and Chic1, and there are the same number of CTCF sites within each locus. One difference in the organisation of these sites is the spacing between them: CTCF sites within Chic1 are more clustered than the ones within Linx - this could potentially play a role in orchestrating which and how contacts are formed. "Loop extrusion" has been proposed as a mechanism to form TADs and chromatin contacts (Fudenberg et al., 2016; Goloborodko et al., 2016; Sanborn et al., 2015), by which an "extruding factor" (such as cohesin) engulfs two DNA chains and moves along them, extruding DNA until it reaches "stalling factors" (such as CTCF), which block its progression; a chromatin loop would thus be formed and stabilized. Could the length of the intervals between CTCF sites influence the likelihood at which the cohesin complex (or the extruding factor) gets stalled? Perhaps more distributed sites (like at the Linx locus) rather than more clustered (like at the Chic1 locus) provide more opportunities for stalling cohesin, given the very fast rate at which CTCF binds and unbinds chromatin (Hansen et al., 2017) and the rate of extrusion by cohesin (Davidson et al., 2019; Kim et al., 2019). Another potential explanation (not mutually exclusive) is that the differences in "contact potential" depend on the different sequences flanking the consensus CTCF motifs within Linx and Chic1, as suggested by a recent study on CTCF sites as transcriptional insulators (Huang et al., 2021).

Transcriptional changes in mutant $245 \mathrm{~kb}-\mathrm{INV}$ mESCs and during differentiation, when compared to control, were observed for two genes (Nap1L2 and TsX) within the Tsix-TAD (Fig. 3). As discussed previously, we think that these changes are associated with (genomic) proximity to the enhancer element Xite and not necessarily with the new topological structure of the inverted Tsix-TAD. Perhaps more interesting is the fact that most other genes within the Tsix-TAD do not show changes in expression, especially the Linx, Chic1 and Xite loci, which are involved in the topological changes observed for the $245 \mathrm{~kb}-\mathrm{INV}$ allele. This could 
have a number of explanations: (i) the expression of these genes might not be particularly reliant on cis-regulation, and therefore impervious to topological changes; (ii) interactions between these genes and their cis-regulatory elements might not depend on topological organisation, and therefore still occur regardless of the topological changes; or (iii) interactions between these genes and their cis-regulatory elements might depend on topological organisation, and the new contacts allow these interactions to occur as efficiently as in wild type, and therefore no changes in expression are observed. Further genetic exploration of these loci will be critical to exclude hypotheses.

Surprisingly, expression of Xist, which lies outside of the Tsix-TAD, in the neighbouring TAD, was mildly upregulated, to an extent that we could detect accumulation of Xist RNA in "clouds" in mutant male cells, which we never observe(d) in wild type male cells (Fig. 3). This upregulation could be associated with one or more of the other alterations observed on the $245 \mathrm{~kb}$-INV allele, either structural, or transcriptional, or both. For instance, we observed reduced expression for Tsix, Xist's antisense cis-repressor, in the pluripotent state, which could have an impact in Xist regulation; during differentiation, however, when Xist is upregulated, we did not detect differences in Tsix expression. Further research will be needed to clarify the involvement of Tsix in the Xist phenotype observed here.

Could Xist upregulation be a consequence of Nap1L2 upregulation or TsX downregulation? Genetic studies with Nap1L2 (Attia et al., 2007; Rogner et al., 2000) did not report any effects on Xist expression or sex-specific phenotypes; upregulation of Nap1L2 is thus unlikely to cause Xist upregulation, although this cannot be formally excluded. On the other hand, knockout studies of Tsx (Anguera et al., 2011) reported Xist RNA clouds in a small percentage of differentiating male mutant mESCs; the authors proposed that Xist expression was upregulated due to its negative cis-regulators Tsix and Xite being downregulated during differentiation. This Xist phenotype is identical to the one we observed (Fig. 3), although in $245 \mathrm{~kb}$-INV mutant cells there is still some Ts $x$ expression (contrary to the Tsx knockout) and we did not observe changes in Tsix or Xite expression during differentiation. Downregulation of TsX in 245kb-INV mutant cells might thus account, partially or maybe even completely, to ectopic Xist upregulation. This raises interesting questions of how such inter-TAD regulation/communication between TsX and Xist could occur. Similarly, we have recently reported that another locus within the Tsix-TAD, Linx, contains sequences that affect expression of Xist in the neighbouring TAD in a Tsix-independent manner (Galupa et al., 2020). A slight increase in Xist expression in cis was also observed in $245 \mathrm{~kb}-\mathrm{INV}$ heterozygous embryos, but it was not statistically significant and did not result in skewed patterns of X-inactivation (Fig. 4). These results underlie the importance of verifying whether changes in gene expression result or not in differences of the phenotypes they mediate - in many studies it often remains an open question whether the changes observed in gene expression, especially when modest, do matter for the processes in which those genes are involved.

In agreement with previous studies, our study illustrates that the relationship between chromosome structure and gene expression is rather complex. The almost "dogmatic" view that TADs restrict gene cis-regulation (Finn and Misteli, 2019; Koch, 2019) is at odds with a 
growing amount of evidence that mechanisms of inter-TAD communication exist, albeit potentially subject to modulation by TADs and their boundaries. Here, we showed that, on the one hand, expression of genes within a TAD can be quite tolerant to changes in contacts within that TAD; on the other hand, we also showed that inversion of a large region within a $\mathrm{TAD}$ affected the expression of a gene in the neighbouring TAD, potentially due to accompanying changes in topological organisation and topological insulation. Further investigations are warranted for a more complete understanding of the relationship between the topological organisation of the genome and the transcriptional regulation of its genes.

\section{Materials and methods}

All the materials and methods described below have also been published previously (Galupa et al., 2020).

\section{Tissue culture conditions}

E14 mESC line and clones derived from it were grown on flasks or dishes coated with $0.1 \%$ (wt/vol) gelatin. Culture media for mESC consisted in Glasgow medium (Gibco) supplemented with $2 \mathrm{mM}$ L-Glutamine, $0.1 \mathrm{mM}$ nonessential amino acids, $1 \mathrm{mM}$ sodium pyruvate, 15\% FBS (Gibco), $0.1 \mathrm{mM}$ b-mercaptoethanol (Sigma) and $1000 \mathrm{U} / \mathrm{mL}$ of LIF (Chemicon). All lines were cultivated at $37^{\circ} \mathrm{C}$ under $8 \% \mathrm{CO}_{2}$ and passaged according to their confluency, generally every other day. Medium was refreshed daily. For early differentiation assays, mESC were washed with $1 \mathrm{x}$ PBS, incubated with trypsin at $37^{\circ} \mathrm{C}$ for $20 \mathrm{~min}$ and resuspended in ES medium without LIF. After cell counting, desired number of cells was resuspended in differentiation medium and $8^{*} 10^{5}$ cells per well were seeded in a fibronectincoated (10 $\square \mathrm{g} / \mathrm{mL}$, Millipore) 6-well plate in differentiation medium. Differentiation medium was consisted of N2B27 medium, $20 \mathrm{ng} / \mathrm{mL}$ activin $\mathrm{A}(\mathrm{R} \& \mathrm{D})$ and $12 \mathrm{ng} / \mathrm{mL}$ FGF-basic (R\&D). Differentiation medium was changed daily and cells were washed in PBS before collection to remove dead cells.

\section{Mouse experimentation}

Animal care and use for this study were performed in accordance with the recommendations of the European Community (2010/63/UE) for the care and use of laboratory animals. Experimental procedures, including genomic engineering (see below), are in compliance with international guidelines and were specifically approved by the ethics committee of the Institut Curie CEEA-IC \#118 and given authorization by the French national authorities (references: APAFIS\#\#13962-2018030717538778-v2 and APAFIS\#8812-2017020611033784-v2).

Postimplantation embryos were collected at E8.5-10.5 stages, assuming plugging at midnight. Females with a vaginal plug were weighted every other day and only taken for dissection if a significant increase in weight was observed $(\sim 2 \mathrm{~g}$ for B6D2F1 mice, $\sim 1 \mathrm{~g}$ for JF1 mice) at expected time of E8.5-E10.5 development. Extraembryonic tissues were taken for sexing the embryos. Whole embryo proper was washed three times in 1xPBS before frozen for allelic expression analysis. 
Genomic engineering of mice and mESC

Inversion $245 \mathrm{~kb}-\mathrm{INV}$ and deletion Chic1-14kb $\Delta$ were generated using CRISPR-Cas9 (mESC and mice) technologies, and the process is described below. Inversions within the Linx locus (Linx-25kb-INV and Linx-51kb-INV) were generated using the same constructs and primers as the equivalent deletions, which were described in (Galupa et al., 2020). The deletion Chic1-4kb $\Delta$ was previously generated (Giorgetti et al., 2014).

We designed sgRNAs to flank the region of interest:

- For 245kb-INV: CR30 (ACTGGTTCAGCCACTCACCG) and CR32 (CTGAGCTGGTTCATACAGGT)

- For Chic1-14kb $\Delta$ : CR21 (AAAGATCGTTTCTATCTAGC) and CR16R (CGCCAAACTTCCAAAATGGC)

For cloning sgRNAs, we used pX459-v2 (Plasmid \#62988; Addgene) and protocol from the Zhang lab (https://media.addgene.org/cms/filer_public/e6/5a/e65a9ef8-c8ac-4f88-98da3b7d7960394c/zhang-lab-general-cloning-protocol.pdf). sgRNA constructs were amplified upon transformation of $\mathrm{DH} 5 \alpha$ competent cells (Takara) grown at $37^{\circ} \mathrm{C}$, and sequenced for verifying correct cloning. Midipreps for all constructs were prepared at final concentration $>1 \mathrm{mg} / \mathrm{mL}$ using the NucleoBond Xtra Midi Plus kit (Macherey-Nagel).

mESC were transfected with sgRNA constructs using the P3 Primary Cell 4D-Nucleofector X Kit (V4XP-3024) and the Amaxa 4D Nucleofector ${ }^{\mathrm{TM}}$ system (Lonza). We used the transfection programme CG-104. Each transfection included 5 million cells resuspended in the nucleofection mix (prepared according to manufacturer's instructions) containing $5 \mu \mathrm{g}$ of each sgRNA (two constructs). As a transfection control, 10 $\mu \mathrm{g}$ of pmaxGFP (Lonza) were used, for which the nucleofection efficiency was around $90 \%$. Cells were immediately resuspended in pre-warmed culture medium after nucleofection and seeded at three serial 10x dilutions in 10-cm dishes to ensure optimal density for colony-picking. Transfected cells were selected with puromycin for $48 \mathrm{~h}$, and grown for 8-10 days. Single colonies were picked into 96-well plates. Genomic DNA was isolated in 96-well plates for PCR-based screening of inversions. Genotyping primers:

- For 245kb-INV: RG82 (CAATCACTCTTGCCTTACCAATT), RG83 (CCCAAACCAACCCTTGACTG), RG84 (GTTGGGACCTAAACTCTAGTACA) and RG85 (AGTGGACTAGCTTTGCCTCA)

- For Chic1-14kbs: EN118 (GCCTGCAGTCTTACCAGGAG), EN119 (TAATCTGCAGCGTGTTGAGG), RG123 (TCCTCCCTTACCAGTCTCCT), RG124 (CAGAATCCCGGATGTGAGGA)

The strategy was inspired on the Epigenesys protocol by Nora and Heard, 2012, described in:

https://www.epigenesys.eu/en/protocols/genome-engineering/816-engineering-genomicdeletions-and-inversions-in-mouse-es-cells-using-custom-designed-nucleases.

We sequenced the PCR products from the inversion alleles to determine the breakpoints: 
- For 245kb-INV - clone1: chrX-100377328 and chrX-100622017; clone2: chrX100377337 and chrX-100622025 (coordinates in mm9)

- For Chic1-14kb $\Delta$ - clone1: chrX-103370850 and chrX-103384956 (coordinates mm10)

The mouse mutant lines were generated following the strategy described in (Wang et al., 2013) with minor modifications. Cas 9 mRNA was in vitro transcribed from a T7-Cas9 pCR2.1-XL plasmid (Greenberg et al., 2017) using the mMESSAGE mMACHINE T7 ULTRA kit (Life Technologies) and purified with the RNeasy Mini kit (Qiagen), or bought from Tebu-bio (L-7206). The sgRNAs were amplified by PCR with primers containing a 5' $\mathrm{T} 7$ promoter sequence from the plasmids used for mESC transfection. After gel purification, the T7-sgRNA PCR products were used as the template for in vitro transcription with the MEGAshortscript T7 kit (Life Technologies) and the products were purified using the MEGAclear kit (Life Technologies). Cas9 mRNA and the sgRNAs were eluted in DEPCtreated RNase-free water, and their quality was assessed by electrophoresis on an agarose gel after incubation at $95^{\circ} \mathrm{C}$ for $3 \mathrm{~min}$ with denaturing agent provided with the in vitro transcription kits. Cas9 mRNA and sgRNAs (at $100 \mathrm{ng} / \mu 1$ and $50 \mathrm{ng} / \mu \mathrm{l}$, respectively) were injected into the cytoplasm of mouse B6D2F1 zygotes from eight-week-old superovulated B6D2F1 (C57BL/6J $\times$ DBA2) females mated to stud males of the same background. Zygotes with well-recognized pronuclei were collected in M2 medium (Sigma) at E0.5. Injected embryos were cultured in M16 medium (Sigma) at $37^{\circ} \mathrm{C}$ under $5 \% \mathrm{CO} 2$, until transfer at the one-cell stage the same day or at the two-cell stage the following day to the infudibulum of the oviduct of a pseudogestant CD1 female at E0.5 (25-30 embryos were transferred per female). All weaned mice (N0) were genotyped for presence of inversion alleles using the same genotyping primers as for mESC mutant lines. Mice carrying inversion alleles were crossed to B6D2F1 mice and their progeny screened again for the presence of the inversion allele. The F1 mice were considered the "founders" and bred to B6D2F1 mice; their progeny was then intercrossed to generate homozygous mice and lines were kept in homozygosity.

\section{RNA fluorescent in situ hybridisation (FISH)}

RNA FISH was performed as described previously with minor modifications (Chaumeil et al., 2008). Briefly, differentiating mESCs were dissociated using accutase (Invitrogen) and adsorbed onto Poly-L-Lysine (Sigma) coated coverslips \#1.5 (1mm) for $5 \mathrm{~min}$. Cells were fixed with 3\% paraformaldehyde in PBS for $10 \mathrm{~min}$ at room temperature and permeabilized for $5 \mathrm{~min}$ on ice in PBS containing 0.5\% Triton X-100 and 2mM Vanadylribonucleoside complex (New England Biolabs). Coverslips were preserved in $70 \% \mathrm{EtOH}$ at $-20^{\circ} \mathrm{C}$. To start FISH experiments, coverslips were dehydrated through an ethanol series $(80 \%, 95 \%$, and $100 \%$ twice) and air-dried quickly, then lowered onto a drop of the probe/hybridization buffer mix $(50 \%$ Formamide, 20\% Dextran sulfate, 2x SSC, $1 \mu \mathrm{g} / \mu \mathrm{l}$ BSA, 10mM Vanadylribonucleoside) and incubated overnight at $37^{\circ} \mathrm{C}$. The next day, coverslips were washed three times at $42{ }^{\circ} \mathrm{C}$ in $50 \%$ formamide in $2 \times \mathrm{SSC}(\mathrm{pH} 7.2-7.4)$ and three times at $42{ }^{\circ} \mathrm{C}$ in $2 \times \mathrm{SSC}$. Nuclei were counterstained with DAPI $(0.2 \mathrm{mg} / \mathrm{ml})$, coverslips were mounted $(90 \%$ glycerol, $0.1 \mathrm{X}$ PBS, $0.1 \%$ p-phenylenediamine at $\mathrm{pH} 9$ ), and cells were imaged using a wide-field DeltaVision Core microscope (Applied Precision). 
Probes used were a Huwe1 bacterial artificial chromosome, BAC (BACPAC Resources Center, RP24-157H12) and oligos ( $\sim 75$ nucleotides long) covering all Xist exons (Roche, custom design). The BAC was labelled using the Nick Translation kit from Abbot and following manufacturer's instructions. Oligos were end-labeled with Alexa488 fluorophore (from manufacturer). Probes were either ethanol-precipitated (BAC) or vacuum-dried (oligos) and resuspended in formamide with shaking at $37^{\circ} \mathrm{C}$. BAC was co-precipitated with mouse Cot-1 DNA (Invitrogen), and competition to block repetitive sequences was performed for at least $20 \mathrm{~min}$ at $37^{\circ} \mathrm{C}$, and after denaturation $\left(75^{\circ} \mathrm{C}, 10 \mathrm{~min}\right)$. Probes were then mixed with one volume of $2 \times$ hybridization buffer.

Gene expression analysis ( $m E S C s$ )

Cells were collected for gene expression analysis at $0 \mathrm{~h}, 12 \mathrm{~h}, 24 \mathrm{~h}, 36 \mathrm{~h}, 48 \mathrm{~h}$ and $60 \mathrm{~h}$ of differentiation. Cells were lysed with Trizol (Invitrogen), and RNA was isolated using the RNAeasy Mini kit (Qiagen), including DNase treatment. RNA samples were systematically run on an agarose gel to check their integrity. For reverse transcription cDNA was synthesised from $0.5 \mu$ g of RNA using SuperScript ${ }^{\mathrm{TM}}$ III Reverse Transcriptase and random primers (both Invitrogen) according to the manufacturer's recommendations. Two independent reverse transcription experiments were carried out for each sample, pooled at the end and diluted 25-fold prior to qPCR or allelic expression analysis. No-reverse transcription controls were processed in parallel. We used the NanoString nCounter gene expression system (Geiss et al., 2008) to systematically characterise transcriptional differences in wildtype and mutant mESC, prior or during differentiation. We used 500ng of total RNA from each sample for each nCounter hybridization round. We designed a customised probe codeset (van Bemmel et al., 2019) to identify nearly a hundred transcripts from Xic genes, other X-linked genes, pluripotency factors, differentiation markers, proliferation markers and normalization genes. Standard positive controls included in the kit were used for scaling the raw data. Genes Actb, Rrm2 and Sdha were used for normalization. Differential expression was always calculated for samples run on the same nCounter hybridization.

\section{Allelic expression analysis (mouse embryos)}

Embryos were lysed in RLT buffer (Qiagen) supplemented with 0.01\% 2-mercaptoethanol, and after two rounds of vortexing (15sec each), lysates were applied directly to a QIAshredder spin column (Qiagen) and centrifuged for $3 \mathrm{~min}$ at full speed. RNA was extracted using the RNAeasy Mini kit (Qiagen), including DNase treatment, and following manufacturer's instructions. RNA samples were systematically run on an agarose gel to check their integrity. cDNA was prepared as described above for gene expression analysis for mESCs, and then PCR-amplified with biotinylated primers and pyrosequenced for allele quantification on a Pyromark Q24 system (Qiagen). The same PCR was done on no-reverse transcription control samples to confirm absence of genomic DNA contamination. Primers used were designed using the PyroMark Assay Design software and validated on XX polymorphic genomic DNA for a ratio of 50:50\% ( $\pm 4 \%)$. List of primers and SNPs used for allele quantification can be found in (Galupa et al., 2020).

\section{Chromosome conformation capture}


3C libraries were prepared based on previous protocols (Nora et al., 2017; Rao et al., 2014b), with some modifications. Crosslinked cells (in 2\% Formaldehyde; 10 million for each sample) were lysed in $10 \mathrm{mM}$ Tris- $\mathrm{HCl}, \mathrm{pH} 8,10 \mathrm{mM} \mathrm{NaCl}, 0.2 \% \mathrm{NP}-40,1 \times$ complete protease inhibitor cocktail (Roche) for $15 \mathrm{~min}$ on ice. Nuclei were resuspended in $100 \mu \mathrm{L}$ $0.5 \% \mathrm{SDS}$, incubated at $62^{\circ} \mathrm{C}$ for $10 \mathrm{~min}$ and quenched with $50 \mu \mathrm{L} 10 \%$ Triton X-100 and 290 $\mu \mathrm{L}$ water at $37^{\circ} \mathrm{C}$ for $15 \mathrm{~min}$. Digestion was performed overnight by adding $50 \mu \mathrm{L}$ of DpnII (Capture-C) or HindIII (5C) buffer and $10 \mu \mathrm{L}$ of high-concentration DpnII or HindIII (NEB) and incubating samples at $37^{\circ} \mathrm{C}$ in a thermomixer. Before this step, an aliquot was taken from each sample as an undigested control. Digests were heat inactivated for $20 \mathrm{~min}$ at $65^{\circ} \mathrm{C}$ and an aliquot was taken from each sample as a digested (unligated) control. Samples were cooled at room temperature for 10 min before adding the ligation cocktail. 3C libraries were ligated for 4 hours at $25^{\circ} \mathrm{C}$ with 10U T4 ligase and ligation buffer (ThermoFisher cat 15224) in a thermomixer at 1000rpm. Ligated samples were then centrifuged at 2000rpm, resuspended in $240 \mu \mathrm{L}$ of $5 \%$ SDS and $1 \mathrm{mg}$ Proteinase $\mathrm{K}$, incubated at $55^{\circ} \mathrm{C}$ for $30 \mathrm{~min}$, supplemented with $50 \mu \mathrm{L} 5 \mathrm{M} \mathrm{NaCl}$ and incubated at $65^{\circ} \mathrm{C}$ for 4 hours. DNA was then purified by adding $500 \mu \mathrm{L}$ isopropanol, incubated at $-80^{\circ} \mathrm{C}$ overnight, centrifuged at 12,000 $\mathrm{rpm}$ at $4^{\circ} \mathrm{C}$, washed with $70 \%$ ethanol, air dried and resuspended in $100 \mu \mathrm{L}$ water, followed by incubation with RNase A at $37^{\circ} \mathrm{C}$ for one hour. $3 \mathrm{C}$ templates were quantified using Qubit DNA Broad-Range (ThermoFisher) and diluted to $100 \mathrm{ng} / \mu \mathrm{L}$. Libraries and respective controls (undigested and digested aliquots) were verified on a gel.

5C was performed as described in (Nora et al., 2017), which adopts a single-PCR strategy to construct 5C-sequencing libraries from the $3 \mathrm{C}$ template. Briefly, four $10 \mu \mathrm{L} 5 \mathrm{C}$ annealing reactions were assembled in parallel, each using $500 \mathrm{ng}$ of $3 \mathrm{C}$ template, $1 \mu \mathrm{g}$ salmon sperm (ThermoFisher) and $10 \mathrm{fmol}$ of each $5 \mathrm{C}$ oligonucleotide in $1 \mathrm{X}$ NEBuffer ${ }^{\mathrm{TM}} 4$ (5C set of oligonucleotides described in Nora et al., 2012). Samples were denatured at $95^{\circ} \mathrm{C}$ for 5 min and incubated at $48^{\circ} \mathrm{C}$ for $16-18 \mathrm{~h} .10 \mu \mathrm{L}$ of $1 \mathrm{X}$ Taq ligase buffer with $5 \mathrm{U}$ Taq ligase were added to each annealing reaction followed by incubation at $48^{\circ} \mathrm{C}$ for $4 \mathrm{~h}$ and $65^{\circ} \mathrm{C}$ for $10 \mathrm{~min}$. Negative controls (no ligase, no template or no $5 \mathrm{C}$ oligonucleotide) were included during each experiment to ensure the absence of contamination. To attach Illumina-compatible sequences, 5C libraries were directly PCR amplified with primers harboring 50-mer tails containing Illumina sequences that anneal to the universal T3/T7 portion of the 5C oligonucleotides (Nora et al., 2017). For this, each 5C ligation reaction was used as the template for three parallel PCRs (12 PCRs total), using per reaction $6 \mu \mathrm{L}$ of $5 \mathrm{C}$ ligation with 1.125 U AmpliTaq Gold (ThermoFisher) in 1X PCR buffer II, $1.8 \mathrm{mM} \mathrm{MgCl}, 0.2 \mathrm{mM}$ dNTPs, $1.25 \mathrm{mM}$ primers in $25 \mathrm{~mL}$ total. Cycling conditions were $95^{\circ} \mathrm{C}$ for $9 \mathrm{~min}, 25$ cycles of $95^{\circ} \mathrm{C}$ for $30 \mathrm{sec}, 60^{\circ} \mathrm{C}$ for $30 \mathrm{sec}, 72^{\circ} \mathrm{C}$ for $30 \mathrm{sec}$ followed by $72^{\circ} \mathrm{C}$ for $8 \mathrm{~min}$. PCR products from the same $3 \mathrm{C}$ sample were pooled and run on a $2.0 \%$ agarose electrophoresis gel. 5C libraries $(231 \mathrm{bp}$ ) were then excised and purified with the MinElute Gel Extraction kit (QIAGEN). Library concentrations were estimated using TapeStation (Agilent) and Qubit (ThermoFisher), pooled and sequenced using $12 \mathrm{pM}$ for the loading on rapid flow cells using the HiSeq 2500 system (Illumina). Sequencing mode was set as 20 dark cycles followed by 80 bases in single end reads (SR80). 
Sequencing data was processed using our custom pipeline, 5C-Pro, available at https://github.com/bioinfo-pf-curie/5C-Pro. Briefly, single-end sequencing reads were first trimmed to remove Illumina adapters and aligned on an in silico reference of all pairs of forward and reverse primers using the bowtie2 software (Langmead and Salzberg, 2012). Aligned reads were then directly used to infer the number of contacts between pairs of forward and reverse primers, thus providing a 5C map at the primer resolution. Based on our previous experiments, inefficient primers were discarded from downstream analysis. Quality controls of the experiments were then performed using the HiTC BioConductor package (Servant et al., 2012). Data from biological replicates were pooled (summed) and binned using a running median (window $=30 \mathrm{~kb}$, final resolution $=6 \mathrm{~kb}$ ). We normalized $5 \mathrm{C}$ contacts for the total number of reads and filtered out outlier probes and singletons, as previously described (Hnisz et al., 2016; Nora et al., 2012; Smith et al., 2016). We also developed a novel method to exclude noisy contacts in the $5 \mathrm{C}$ maps, called "neighbourhood coefficient of variation", available at https://github.com/zhanyinx/Coefficient_Variation. Considering that the chromatin fiber behaves as a polymer, the contact frequency of a given pair of genomic loci (e.g. $i$ and $j$ ) cannot be very different from those of fragments $i \pm N$ and $j \pm N$ if $N$ is smaller (or in the order of) than the persistence length of the chromatin fiber. Hence, a given pixel in the 5C map (which is proportional to the contact frequency between the two corresponding loci) can be defined as noisy if its numerical value is too different from those corresponding to neighboring interaction frequencies. To operatively assess the similarity of a given interaction with neighboring contacts, we calculated the coefficient of variation $(\mathrm{CV})$ of contacts (pixels in the 5C map) in a 10x10 square centered on every contact. We then set out to discard pixels for which the corresponding coefficient of variation was bigger than a threshold. Given that the distribution of the coefficient of variation of all $5 \mathrm{C}$ samples in this study is bimodal around $\mathrm{CV}=1$, we set the $\mathrm{CV}$ threshold to 1 . Discarded contacts appear as grey pixels in the differential 5C maps. For differential analysis between two samples of interest, we calculated the difference between Z-scores determined for each individual map (Smith et al., 2016). Samples corresponding to inversions of genomic regions were mapped to a virtually inverted map before analysis. Samples corresponding to deletions were corrected for the new distance between genomic elements; this distance-adjustment was performed along with the Z-score calculation. 5C data for E14 cell line (used as control) has been published previously (Galupa et al., 2020) but control and mutant samples were collected and processed in parallel.

\section{Statistical analysis}

For RNA FISH, nCounter and allelic expression analysis, statistical details of experiments can be found in the figure legends, figures and/or Results, including the statistical tests used, exact value of $\mathrm{n}$ and what $\mathrm{n}$ represents.

\section{Accession numbers}

All next-generation sequencing data generated in this study has been deposited in the Gene Expression Omnibus (GEO) under the accession number GSE124596 (5C data for E14 cell line) and GSE180617 (5C data for 245kb-INV cell line). 5C data for E14 cell line (used as 
control) has been published previously (Galupa et al., 2020) but control and mutant samples were collected and processed in parallel.

To review GEO accession GSE180617:

Go to https://www.ncbi.nlm.nih.gov/geo/query/acc.cgi?acc=GSE180617

Enter token gdelimmatputvod into the box

\section{Competing interest statement}

The authors declare no competing interests.

\section{Acknowledgments}

We are grateful to Isabelle Grandjean for help and advice with animal management; to Patricia Diabangouaya for help with mouse genotyping; to Chris Gard for help with gene expression analysis; to Denis Krndija for critical reading of the manuscript. We thank all members of the Heard lab for advice, support, and helpful comments and discussions. We are also thankful to facilities at the Institut Curie, including the Mouse Facility, the BDD team of PICT-IBiSA, the ICGex NGS platform (in particular Sónia Lameiras and Sylvain Baulande), the Genomics Platform (in particular David Gentien, Cécile Reyes, Audrey Rapinat and Benoit Albaud) and the Bioinformatics Platform. We acknowledge the Zhang lab for sharing plasmids, and the ENCODE Consortium and the Bruneau, Ren, Sharp, Stamatoyannopoulos and Young labs for generating datasets used in this study. Funding: This work was supported by fellowships from Région Ile-de-France (DIM Biothérapies) and Fondation pour la Recherche Médicale (FDT20160435295) to RG; ERC Advanced Investigator award (ERC2014-AdG no. 671027), Labelisation La Ligue, FRM (DEI20151234398), ANR DoseX 2017, Labex DEEP (ANR-11-LBX-0044), part of the IDEX PSL (ANR-10-IDEX-0001-02 PSL) and ABS4NGS (ANR-11-BINF-0001) to EH.

\section{Author contributions}

Conceptualization: RG, LG, EH. Investigation: RG, CP, EPN. Methodology: RG, CP, EPN, FEM, CJ, MB, KA. Formal analysis: RG, NS, YZ, JvB. Data curation: RG, NS, YZ, JvB, LG. Visualization: RG, YZ. Software: NS, YZ, JvB, LG. Supervision: RG, MB, KA, LG, EH. Project administration: RG, EH. Funding acquisition: EH. Writing, original draft: RG. Writing, review \& editing: RG, KA, LG, EH.

\section{Figure legends}


Figure 1. Strategy for inverting the nearly entire Tsix-TAD. (A) Topological organisation of the Xic (top) and chromatin ChIP-seq profiles (bottom; see Methods for sources); the Xist/Tsix locus lies at the boundary between two TADs. (B) Targeting strategy for inverting the $\sim 245 \mathrm{~kb}$ region comprising most of the Tsix-TAD, except Tsix and its known regulator Xite, and leaving the boundaries intact. (C) PCR strategy (bottom) and gel results (top) for detecting the inversion events. E14 is the wild type, parental cell line. $\mathrm{Cl} .1$ and $\mathrm{Cl} .2$ are the two clones that were generated and analysed throughout the study.

Figure 2. Rearrangement of contacts within the TAD and increased insulation with neighbouring TAD upon $245 \mathrm{~kb}$ intra-TAD inversion. (A) $5 \mathrm{C}$ profiles of wildtype (WT; two replicates pooled) and $245 \mathrm{~kb}-\mathrm{INV}$ mutant (two clones pooled) mESCs. Mutant map is corrected for inversion and grey pixels represent filtered contacts (see Methods). (B) $(C)$ Zoom-ins of the maps in (A) and 5C differential maps, representing the subtraction of Zscores calculated for wildtype and $245 \mathrm{~kb}-\mathrm{INV}$ mutant maps separately. Grey pixels represent filtered contacts. (D) Insulation scores across the Xic TADs and downstream TADs based on $5 \mathrm{C}$ profiles for wildtype and $245 \mathrm{~kb}-\mathrm{INV}$ mutant mESCs. The "valleys" represent TAD boundaries.

Figure 3. Inversion leads to transcriptional changes of specific genes within the TAD and of Xist across the TAD boundary. (A) Schematic representation of mESC to EpiLSC differentiation and time points analysed by Nanostring nCounter (see Methods). (B) Gene expression analysis during differentiation. Data is normalised to wt-d0 for each gene, and represents the average of two biological replicates (wild type) or of two independent clones (mutant). Statistical analysis: two-tailed paired t-test $(* \mathrm{p}<0.05 ; * * \mathrm{p}<0.01 ; * * * \mathrm{p}<0.001)$. (C) RNA FISH for Huwe1 (X-linked gene outside of the Xic) and Xist (exonic probe) on mESCs differentiated to d1.5. Percentage of cells with Xist RNA accumulation is indicated and represents an average from two independent experiments. Scale bar: $2 \square \mathrm{m}$.

Figure 4. Female embryos with $245 \mathrm{~kb}-\mathrm{INV}$ allele show a bias in Xist expression. $(A, D)$ Crosses used for analysis of RNA allelic ratios in female hybrid embryos inheriting the $M$. musculus domesticus allele paternally $(A)$ or maternally $(D)$. Tables summarise number of embryos collected. ( $B, C, E, F)$ RNA allelic ratios for the X-linked gene Atp7a $(B, E)$ and Xist $(C, F)$. Each black dot corresponds to a single female embryo. Statistical analysis was performed using Mann-Whitney test (see Methods).

Figure 5. Inversion of Linx cluster of CTCF sites leads to Xist upregulation in cis. (A) The Linx locus, CTCF binding, and orientation of CTCF motifs associated with CTCF chromatin immunoprecipitation sequencing (ChIP-seq) peaks. The targeted inversions Linx-25kb-INV and Linx-51kb-INV are indicated. (B) 5C profiles (Tsix-TAD zoom-in) of wildtype (WT; two replicates pooled) and Linx-51kb-INV (two clones pooled) mESCs, and 5C differential map, representing the subtraction of Z-scores calculated for wildtype and Linx-51kb-INV maps. (C) Left, 5C profile of Linx-51kb-INV mESCs (two clones pooled); map is corrected for inversion and grey pixels represent filtered contacts (see Methods). Right, 5C differential map, representing the subtraction of Z-scores calculated for wildtype and Linx-51kb-INV maps separately. (D) Insulation scores across the Xic TADs and downstream TADs based on 
5C profiles for wildtype and Linx-51kb-INV mESCs. The "valleys" represent TAD boundaries. (E) Gene expression analysis during differentiation. Data is normalised to wt-d0 for each gene, and represents the average of two biological replicates (wild type) or of two independent clones (mutant). Statistical analysis: two-tailed paired t-test (all nonsignificant). $(F, G)$ Left, crosses used for analysis of RNA allelic ratios in female hybrid embryos inheriting the $M$. musculus domesticus allele paternally $(F)$ or maternally $(G)$. Tables summarise number of embryos collected. Right, RNA allelic ratios for Xist and the X-linked gene Atp7a. Each black dot corresponds to a single female embryo. Statistical analysis was performed using Mann-Whitney test $(* \mathrm{p}<0.05)$.

Figure 6. Deletion of Chic1 cluster of CTCF sites leads to Xist downregulation in cis. $(A)$ The Chic1 locus, CTCF binding, and orientation of CTCF motifs associated with CTCF chromatin immunoprecipitation sequencing (ChIP-seq) peaks. The targeted deletions Chic1$4 \mathrm{~kb} \Delta$ and Chic $1-14 \mathrm{~kb} \Delta$ are indicated. $(B, C)$ Top, $5 \mathrm{C}$ profiles of Chic $1-4 \mathrm{~kb} \Delta$ ( $B$, two clones pooled) and Chic1-14kb $\Delta$, one clone, two replicates pooled). Middle, 5C differential maps, representing the subtraction of Z-scores calculated for wildtype and deletion maps. Grey pixels represent filtered contacts (see Methods). Bottom, Tsix-TAD zoom-in of differential maps. $(D, E)$ Gene expression analysis during differentiation. Data is normalised to wt-d0 for each gene, and represents the average of two biological replicates (wild type and Chic1-14kb $\Delta$ ) or of two independent clones (Chic1-4kb $\Delta$ ). Statistical analysis: two-tailed paired t-test $(* \mathrm{p}<0.05 ; * * \mathrm{p}<0.01 ; * * * * \mathrm{p}<0.0001)$. (F) Top, RNA allelic ratios for Xist and the X-linked gene Atp7a. Each black dot corresponds to a single female embryo. Statistical analysis was performed using Mann-Whitney test $(* * \mathrm{p}<0.01 ; * * * \mathrm{p}<0.001 ; * * * * \mathrm{p}<0.0001)$. Bottom, crosses used for analysis of RNA allelic ratios in female hybrid embryos inheriting the M. musculus domesticus allele paternally. Tables summarise number of embryos collected.

Figure S1. (A) Virtual 4C plots for the wild type (WT) and 245kb-INV alleles, for which the anchor is the element Xite. The interaction frequency between Xite-TsX is $\sim 5$-fold lower in the inverted allele ( $\sim 50$ counts) than in the WT ( $\sim 250$ counts, and within the region where contact frequency is dominated by genomic distance). Inversely, the interaction frequency between Xite-Nap1L2 in the inverted allele ( $\sim 250$ counts) is $\sim 5$-fold higher than in the WT $(\sim 50$ counts). The changes in interaction frequencies between these elements seem thus to reflect the changes in genomic distances for WT and inverted alleles. (A) Virtual 4C plots for the wild type (WT) and deletion alleles Chic1-4kbD and Chic1-14kbD. The interaction frequency between Xite and Linx is increased in the mutant alleles compared to WT.

\section{References}

Amândio, A.R., Lopez-Delisle, L., Bolt, C.C., Mascrez, B., and Duboule, D. (2020). A complex regulatory landscape involved in the development of mammalian external genitals. ELife 9. 
Anguera, M.C., Ma, W., Clift, D., Namekawa, S., Kelleher, R.J., and Lee, J.T. (2011). Tsx Produces a Long Noncoding RNA and Has General Functions in the Germline, Stem Cells, and Brain. PLoS Genetics 7, e1002248.

Attia, M., Rachez, C., De Pauw, A., Avner, P., and Rogner, U.C. (2007). Nap112 Promotes Histone Acetylation Activity during Neuronal Differentiation. Molecular and Cellular Biology 27, 6093-6102.

Augui, S., Filion, G.J., Huart, S., Nora, E., Guggiari, M., Maresca, M., Stewart, A.F., and Heard, E. (2007). Sensing X chromosome pairs before X inactivation via a novel X-pairing region of the Xic. Science (New York, N.Y.) 318, 1632-1636.

Augui, S., Nora, E.P., and Heard, E. (2011a). Regulation of X-chromosome inactivation by the X-inactivation centre. Nature Reviews. Genetics 12, 429-442.

Augui, S., Nora, E.P., and Heard, E. (2011b). Regulation of X-chromosome inactivation by the X-inactivation centre. Nature Reviews. Genetics 12, 429-442.

Barakat, T.S., Gunhanlar, N., Pardo, C.G., Achame, E.M., Ghazvini, M., Boers, R., Kenter, A., Rentmeester, E., Grootegoed, J.A., and Gribnau, J. (2011). RNF12 activates Xist and is essential for X chromosome inactivation. PLoS Genetics 7, e1002001.

Barakat, T.S., Loos, F., van Staveren, S., Myronova, E., Ghazvini, M., Grootegoed, J.A., and Gribnau, J. (2014). The trans-activator RNF12 and cis-acting elements effectuate X chromosome inactivation independent of X-pairing. Molecular Cell 53, 965-978.

van Bemmel, J.G., Galupa, R., Gard, C., Servant, N., Picard, C., Davies, J., Szempruch, A.J., Zhan, Y., Żylicz, J.J., Nora, E.P., et al. (2019). The bipartite TAD organization of the Xinactivation center ensures opposing developmental regulation of Tsix and Xist. Nature Genetics 51, 1024-1034.

Castillo-Davis, C.I., Mekhedov, S.L., Hartl, D.L., Koonin, E. v., and Kondrashov, F.A. (2002). Selection for short introns in highly expressed genes. Nature Genetics 2002 31:4 31, $415-418$.

Chiaromonte, F., Miller, W., and Bouhassira, E.E. (2003). Gene Length and Proximity to Neighbors Affect Genome-Wide Expression Levels. Genome Research 13, 2602-2608.

Davidson, I.F., Bauer, B., Goetz, D., Tang, W., Wutz, G., and Peters, J.M. (2019). DNA loop extrusion by human cohesin. Science 366, 1338-1345.

Dekker, J., and Heard, E. (2015). Structural and Functional Diversity of Topologically Associating Domains. FEBS Letters 589, 2877-2884.

Dekker, J., and Mirny, L. (2016). The 3D Genome as Moderator of Chromosomal Communication. Cell 164, 1110-1121. 
Denker, A., and de Laat, W. (2016). The second decade of 3C technologies: detailed insights into nuclear organization. Genes \& Development 30, 1357-1382.

Despang, A., Schöpflin, R., Franke, M., Ali, S., Jerković, I., Paliou, C., Chan, W.-L., Timmermann, B., Wittler, L., Vingron, M., et al. (2019). Functional dissection of the Sox9Kcnj2 locus identifies nonessential and instructive roles of TAD architecture. Nature Genetics 51, 1263-1271.

de Wit, E., Vos, E.S.M., Holwerda, S.J.B., Valdes-Quezada, C., Verstegen, M.J.A.M., Teunissen, H., Splinter, E., Wijchers, P.J., Krijger, P.H.L., and de Laat, W. (2015). CTCF Binding Polarity Determines Chromatin Looping. Molecular Cell 60, 676-684.

Dixon, J.R., Selvaraj, S., Yue, F., Kim, A., Li, Y., Shen, Y., Hu, M., Liu, J.S., and Ren, B. (2012). Topological domains in mammalian genomes identified by analysis of chromatin interactions. Nature 485, 376-380.

Dixon, J.R., Jung, I., Selvaraj, S., Shen, Y., Antosiewicz-Bourget, J.E., Lee, A.Y., Ye, Z., Kim, A., Rajagopal, N., Xie, W., et al. (2015). Chromatin architecture reorganization during stem cell differentiation. Nature 518, 331-336.

Dostie, J., Richmond, T.A., Arnaout, R.A., Selzer, R.R., Lee, W.L., Honan, T.A., Rubio, E.D., Krumm, A., Lamb, J., Nusbaum, C., et al. (2006). Chromosome Conformation Capture Carbon Copy (5C): a massively parallel solution for mapping interactions between genomic elements. Genome Research 16, 1299-1309.

Finn, E.H., and Misteli, T. (2019). A genome disconnect. Nature Genetics 51, 1205-1206.

Flavahan, W.A., Drier, Y., Liau, B.B., Gillespie, S.M., Venteicher, A.S., StemmerRachamimov, A.O., Suvà, M.L., and Bernstein, B.E. (2016). Insulator dysfunction and oncogene activation in IDH mutant gliomas. Nature 529, 110-114.

Franke, M., Ibrahim, D.M., Andrey, G., Schwarzer, W., Heinrich, V., Sch?pflin, R., Kraft, K., Kempfer, R., Jerkovi?, I., Chan, W.-L., et al. (2016). Formation of new chromatin domains determines pathogenicity of genomic duplications. Nature 538, 265-269.

Fudenberg, G., Imakaev, M., Lu, C., Goloborodko, A., Abdennur, N., and Mirny, L. (2016). Formation of Chromosomal Domains by Loop Extrusion. Cell Reports 15, 2038-2049.

Furlan, G., Hernandez, N.G., Huret, C., Galupa, R., van Bemmel, J.G., Romito, A., Heard, E., Morey, C., and Rougeulle, C. (2018). The Ftx noncoding locus controls X chromosome inactivation independently of its RNA products. Molecular Cell 70, 462-472.

Galupa, R., Nora, E.P., Worsley-Hunt, R., Picard, C., Gard, C., van Bemmel, J.G., Servant, N., Zhan, Y., el Marjou, F., Johanneau, C., et al. (2020). A Conserved Noncoding Locus Regulates Random Monoallelic Xist Expression across a Topological Boundary. Molecular Cell 77, 352-367.e8. 
Geiss, G.K., Bumgarner, R.E., Birditt, B., Dahl, T., Dowidar, N., Dunaway, D.L., Fell, H.P., Ferree, S., George, R.D., Grogan, T., et al. (2008). Direct multiplexed measurement of gene expression with color-coded probe pairs. Nature Biotechnology 26, 317-325.

Giorgetti, L., Galupa, R., Nora, E.P., Piolot, T., Lam, F., Dekker, J., Tiana, G., and Heard, E. (2014). Predictive Polymer Modeling Reveals Coupled Fluctuations in Chromosome Conformation and Transcription. Cell 157, 950-963.

Goloborodko, A., Marko, J.F., and Mirny, L.A. (2016). Chromosome Compaction by Active Loop Extrusion. Biophysical Journal 110, 2162-2168.

Gontan, C., Achame, E.M., Demmers, J., Barakat, T.S., Rentmeester, E., van IJcken, W., Grootegoed, J.A., and Gribnau, J. (2012). RNF12 initiates X-chromosome inactivation by targeting REX1 for degradation. Nature 485, 386-390.

Greenberg, M.V.C., Glaser, J., Borsos, M., Marjou, F. El, Walter, M., Teissandier, A., and Bourc'his, D. (2017). Transient transcription in the early embryo sets an epigenetic state that programs postnatal growth. Nature Genetics 49, 110-118.

Guo, Y., Xu, Q., Canzio, D., Shou, J., Li, J., Gorkin, D., Jung, I., Wu, H., Zhai, Y., Tang, Y., et al. (2015). CRISPR Inversion of CTCF Sites Alters Genome Topology and Enhancer/Promoter Function. Cell 162, 900-910.

Haarhuis, J.H.I., van der Weide, R.H., Blomen, V.A., Yáñez-Cuna, J.O., Amendola, M., van Ruiten, M.S., Krijger, P.H.L., Teunissen, H., Medema, R.H., van Steensel, B., et al. (2017). The Cohesin Release Factor WAPL Restricts Chromatin Loop Extension. Cell 169, 693707.e14.

Hansen, A.S., Pustova, I., Cattoglio, C., Tjian, R., Darzacq, X., Bernstein, BE., Nusbaum, C., Myers, RM., Brown, M., Li, W., et al. (2017). CTCF and cohesin regulate chromatin loop stability with distinct dynamics. ELife 6, 929-934.

Heard, E., Mongelard, F., Arnaud, D., and Avner, P. (1999). Xist yeast artificial chromosome transgenes function as $\mathrm{X}$-inactivation centers only in multicopy arrays and not as single copies. Molecular and Cellular Biology 19, 3156-3166.

Hnisz, D., Weintraub, A.S., Day, D.S., Valton, A.-L., Bak, R.O., Li, C.H., Goldmann, J., Lajoie, B.R., Fan, Z.P., Sigova, A.A., et al. (2016). Activation of proto-oncogenes by disruption of chromosome neighborhoods. Science (New York, N.Y.) 351, 1454-1458.

Huang, H., Zhu, Q., Jussila, A., Han, Y., Bintu, B., Kern, C., Conte, M., Zhang, Y., Bianco, S., Chiariello, A.M., et al. (2021). CTCF mediates dosage- and sequence-context-dependent transcriptional insulation by forming local chromatin domains. Nature Genetics 1-11.

Jonkers, I., Barakat, T.S., Achame, E.M., Monkhorst, K., Kenter, A., Rentmeester, E., Grosveld, F., Grootegoed, J.A., and Gribnau, J. (2009). RNF12 is an X-Encoded dosedependent activator of $\mathrm{X}$ chromosome inactivation. Cell 139, 999-1011. 
Kim, Y., Shi, Z., Zhang, H., Finkelstein, I.J., and Yu, H. (2019). Human cohesin compacts DNA by loop extrusion. Science 366, 1345-1349.

Koch, L. (2019). Toppling TAD tenets. Nature Reviews Genetics 20, 565.

Langmead, B., and Salzberg, S.L. (2012). Fast gapped-read alignment with Bowtie 2. Nature Methods 9, 357-359.

Lee, J.T., and Lu, N. (1999). Targeted mutagenesis of Tsix leads to nonrandom X inactivation. Cell 99, 47-57.

Luikenhuis, S., Wutz, A., and Jaenisch, R. (2001). Antisense Transcription through the Xist Locus Mediates Tsix Function in Embryonic Stem Cells. Molecular and Cellular Biology 21, $8512-8520$.

Lupiáñez, D.G., Kraft, K., Heinrich, V., Krawitz, P., Brancati, F., Klopocki, E., Horn, D., Kayserili, H., Opitz, J.M., Laxova, R., et al. (2015). Disruptions of Topological Chromatin Domains Cause Pathogenic Rewiring of Gene-Enhancer Interactions. Cell 161, 1012-1025.

McCord, R.P., Kaplan, N., and Giorgetti, L. (2020). Chromosome Conformation Capture and Beyond: Toward an Integrative View of Chromosome Structure and Function. Molecular Cell 77, 688-708.

Nora, E.P., Lajoie, B.R., Schulz, E.G., Giorgetti, L., Okamoto, I., Servant, N., Piolot, T., van Berkum, N.L., Meisig, J., Sedat, J., et al. (2012). Spatial partitioning of the regulatory landscape of the X-inactivation centre. Nature 485, 381-385.

Nora, E.P., Goloborodko, A., Valton, A.-L., Gibcus, J.H., Uebersohn, A., Abdennur, N., Dekker, J., Mirny, L.A., and Bruneau, B.G. (2017). Targeted Degradation of CTCF Decouples Local Insulation of Chromosome Domains from Genomic Compartmentalization. Cell 169, 930-944.e22.

Ogawa, Y., and Lee, J.T. (2003). Xite, X-inactivation intergenic transcription elements that regulate the probability of choice. Molecular Cell 11, 731-743.

Paliou, C., Guckelberger, P., Schöpflin, R., Heinrich, V., Esposito, A., Chiariello, A.M., Bianco, S., Annunziatella, C., Helmuth, J., Haas, S., et al. (2019). Preformed chromatin topology assists transcriptional robustness of Shh during limb development. Proceedings of the National Academy of Sciences of the United States of America 116, 12390-12399.

Phillips-Cremins, J.E., Sauria, M.E.G., Sanyal, A., Gerasimova, T.I., Lajoie, B.R., Bell, J.S.K., Ong, C.-T., Hookway, T.A., Guo, C., Sun, Y., et al. (2013). Architectural Protein Subclasses Shape 3D Organization of Genomes during Lineage Commitment. Cell 153, 1281-1295.

Rao, S.S.P., Huntley, M.H., Durand, N.C., Stamenova, E.K., Bochkov, I.D., Robinson, J.T., Sanborn, A.L., Machol, I., Omer, A.D., Lander, E.S., et al. (2014a). A 3D Map of the Human 
Genome at Kilobase Resolution Reveals Principles of Chromatin Looping. Cell 159, 16651680.

Rao, S.S.P., Huntley, M.H., Durand, N.C., Stamenova, E.K., Bochkov, I.D., Robinson, J.T., Sanborn, A.L., Machol, I., Omer, A.D., Lander, E.S., et al. (2014b). A 3D Map of the Human Genome at Kilobase Resolution Reveals Principles of Chromatin Looping. Cell 159, 16651680 .

Rao, S.S.P., Huang, S.-C., Glenn St Hilaire, B., Engreitz, J.M., Perez, E.M., Kieffer-Kwon, K.-R., Sanborn, A.L., Johnstone, S.E., Bascom, G.D., Bochkov, I.D., et al. (2017). Cohesin Loss Eliminates All Loop Domains. Cell 171, 305-320.e24.

Rastan, S. (1982). Timing of X-chromosome inactivation in postimplantation mouse embryos.

Rastan, S., and Brown, S.D. (1990). The search for the mouse X-chromosome inactivation centre. Genetical Research 56, 99-106.

Rodríguez-Carballo, E., Lopez-Delisle, L., Zhan, Y., Fabre, P.J., Beccari, L., El-Idrissi, I., Nguyen Huynh, T.H., Ozadam, H., Dekker, J., and Duboule, D. (2017). The HoxD cluster is a dynamic and resilient TAD boundary controlling the segregation of antagonistic regulatory landscapes. Genes and Development 31, 2264-2281.

Rogner, U.C., Spyropoulos, D.D., Le Novère, N., Changeux, J.P., and Avner, P. (2000). Control of neurulation by the nucleosome assembly protein-1-like 2. Nature Genetics 25 , $431-435$.

Sanborn, A.L., Rao, S.S.P., Huang, S.-C., Durand, N.C., Huntley, M.H., Jewett, A.I., Bochkov, I.D., Chinnappan, D., Cutkosky, A., Li, J., et al. (2015). Chromatin extrusion explains key features of loop and domain formation in wild-type and engineered genomes. Proceedings of the National Academy of Sciences of the United States of America 112, E6456-65.

Schwarzer, W., Abdennur, N., Goloborodko, A., Pekowska, A., Fudenberg, G., Loe-Mie, Y., Fonseca, N.A., Huber, W., H Haering, C., Mirny, L., et al. (2017). Two independent modes of chromatin organization revealed by cohesin removal. Nature 551, 51-56.

Servant, N., Lajoie, B.R., Nora, E.P., Giorgetti, L., Chen, C.-J., Heard, E., Dekker, J., and Barillot, E. (2012). HiTC: exploration of high-throughput "C" experiments. Bioinformatics (Oxford, England) 28, 2843-2844.

Smith, E.M., Lajoie, B.R., Jain, G., and Dekker, J. (2016). Invariant TAD Boundaries Constrain Cell-Type-Specific Looping Interactions between Promoters and Distal Elements around the CFTR Locus. American Journal of Human Genetics 98, 185-201.

Stavropoulos, N., Lu, N., and Lee, J.T. (2001). A functional role for Tsix transcription in blocking Xist RNA accumulation but not in X-chromosome choice. Proceedings of the National Academy of Sciences of the United States of America 98, 10232-10237. 
Symmons, O., Pan, L., Remeseiro, S., Aktas, T., Klein, F., Huber, W., and Spitz, F. (2016). The Shh Topological Domain Facilitates the Action of Remote Enhancers by Reducing the Effects of Genomic Distances. Developmental Cell 39, 529-543.

Szabo, Q., Bantignies, F., and Cavalli, G. (2019). Principles of genome folding into topologically associating domains. Science Advances 5, eaaw1668.

Tang, Z., Luo, O.J., Li, X., Zheng, M., Zhu, J.J., Szalaj, P., Trzaskoma, P., Magalska, A., Wlodarczyk, J., Ruszczycki, B., et al. (2015a). CTCF-Mediated Human 3D Genome Architecture Reveals Chromatin Topology for Transcription. Cell 163, 1611-1627.

Tang, Z., Luo, O.J., Li, X., Zheng, M., Zhu, J.J., Szalaj, P., Trzaskoma, P., Magalska, A., Wlodarczyk, J., Ruszczycki, B., et al. (2015b). CTCF-Mediated Human 3D Genome Architecture Reveals Chromatin Topology for Transcription. Cell 163, 1611-1627.

Tian, D., Sun, S., and Lee, J.T. (2010). The long noncoding RNA, Jpx, is a molecular switch for X chromosome inactivation. Cell 143, 390-403.

Wang, H., Yang, H., Shivalila, C.S., Dawlaty, M.M., Cheng, A.W., Zhang, F., and Jaenisch, R. (2013). One-Step Generation of Mice Carrying Mutations in Multiple Genes by CRISPR/Cas-Mediated Genome Engineering. Cell 153, 910-918.

Williamson, I., Kane, L., Devenney, P.S., Flyamer, I.M., Anderson, E., Kilanowski, F., Hill, R.E., Bickmore, W.A., and Lettice, L.A. (2019). Developmentally regulated Shh expression is robust to TAD perturbations. Development (Cambridge) 146.

Wutz, G., Várnai, C., Nagasaka, K., Cisneros, D.A., Stocsits, R.R., Tang, W., Schoenfelder, S., Jessberger, G., Muhar, M., Hossain, M.J., et al. (2017). Topologically associating domains and chromatin loops depend on cohesin and are regulated by CTCF, WAPL, and PDS5 proteins. The EMBO Journal 36, 3573-3599.

Zhan, Y., Mariani, L., Barozzi, I., Schulz, E.G., Blüthgen, N., Stadler, M., Tiana, G., and Giorgetti, L. (2017). Reciprocal insulation analysis of Hi-C data shows that TADs represent a functionally but not structurally privileged scale in the hierarchical folding of chromosomes. Genome Research 27, 479-490. 